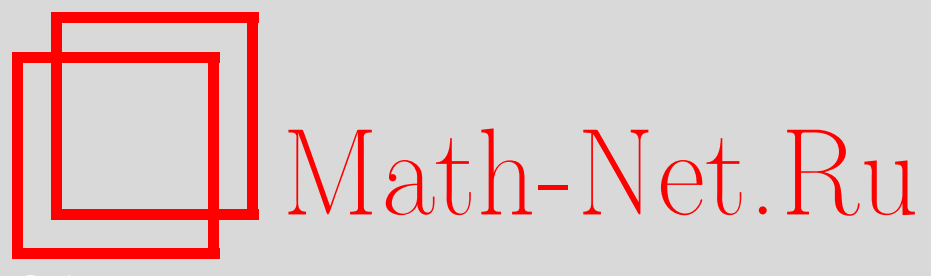

Ю. Ю. Клевцова, О корректности задачи Коши для стохастической системы модели Лоренца бароклинной атмосферы, Матем. сб., 2012, том 203, номер 10, 117-144

DOI: https://doi.org/10.4213/sm7887

Использование Общероссийского математического портала Math-Net.Ru подразумевает, что вы прочитали и согласны с пользовательским соглашением http://www.mathnet.ru/rus/agreement

Параметры загрузки:

IP : 3.89 .185 .249

26 апреля 2023 г., $17: 40: 07$

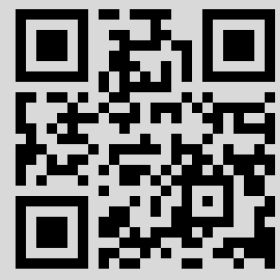




\section{Ю. Ю. Клевцова}

\section{О корректности задачи Коши для стохастической системы модели Лоренца бароклинной атмосферы}

В работе рассматривается задача Коши для одной нелинейной системы дифференциальных уравнений в частных производных с параметрами. Эта система описывает двухслойную квазисоленоидальную модель Лоренца бароклинной атмосферы на вращающейся двумерной сфере. Правая часть системы возмущается белым шумом, рассматриваются случайные начальные данные. Доказывается существование единственного решения и оценка непрерывной зависимости этого решения от совокупности начальных данных и правой части на конечном отрезке времени. Попутно доказывается для задачи Коши с детерминированными начальными данными и правой частью оценка непрерывной зависимости решения от совокупности параметров, начальных данных и правой части на конечном отрезке времени.

Библиография: 32 названия.

Ключевые слова: двухслойная квазисоленоидальная модель Лоренца бароклинной атмосферы, возмущение белым шумом, корректность задачи Коши, случайные начальные данные.

\section{$\S 1$. Введение}

В работе В. Н. Крупчатникова [1] рассматривалась на единичной двумерной сфере $S$ с центром в нуле в сферической системе координат

$$
(\lambda, \varphi), \quad \lambda \in[0,2 \pi), \quad \varphi \in\left[-\frac{\pi}{2}, \frac{\pi}{2}\right], \quad \mu=\sin \varphi,
$$

следующая система уравнений двухслойной квазисоленоидальной модели Лоренца бароклинной атмосферы (физические аспекты вопроса см. в [2]-[4])

$$
\frac{\partial}{\partial t} A_{1} u+\nu A_{2} u+A_{3} u+B(u)=g, \quad t>0
$$

где $\nu>0$ - вязкость, $u(t, x)=\left(u_{1}(t, x), u_{2}(t, x)\right)^{\mathrm{T}}$ - неизвестное векторное поле, $g(t, x)=\left(g_{1}(t, x), g_{2}(t, x)\right)^{\mathrm{T}}$ - детерминированная внешняя сила, $x=(\lambda, \mu)$,

$$
\begin{gathered}
A_{1}=\left(\begin{array}{cc}
-\Delta & 0 \\
0 & -\Delta+\gamma
\end{array}\right), \quad A_{2}=\left(\begin{array}{cc}
\Delta^{2} & 0 \\
0 & \Delta^{2}
\end{array}\right), \\
A_{3}=\left(\begin{array}{cc}
-k \Delta & 2 k \Delta \\
k \Delta & -\left(2 k+k_{1}+\nu \gamma\right) \Delta+\rho
\end{array}\right),
\end{gathered}
$$

$$
B(u)=\left(J\left(\Delta u_{1}+2 \mu, u_{1}\right)+J\left(\Delta u_{2}, u_{2}\right), J\left(\Delta u_{2}-\gamma u_{2}, u_{1}\right)+J\left(\Delta u_{1}+2 \mu, u_{2}\right)\right)^{\mathrm{T}},
$$

Работа выполнена при финансовой поддержке Российского фонда фундаментальных исследований (грант № 12-01-31324). 
здесь параметры $\gamma, \rho, k, k_{1} \geqslant 0$, операторы Якоби $J(\psi, \theta)=\psi_{\lambda} \theta_{\mu}-\psi_{\mu} \theta_{\lambda}$ и Лапласа-Бельтрами (см., например, [5; §1]) $\Delta \psi=\left(\left(1-\mu^{2}\right) \psi_{\mu}\right)_{\mu}+\left(1-\mu^{2}\right)^{-1} \psi_{\lambda \lambda}$ на сфере $S$. Отметим, что константы $\gamma, \rho, k, k_{1}$ неотрицательны, а $\nu$ положительна. В работе [1] было показано, что существует единственное обобщенное решение задачи Коши для этой системы уравнений в некоторых функциональных пространствах. Вопросы разрешимости подобного типа систем рассматривались также в работах [4], [6], и в [6; гл. II, §3] см. ссылки в замечании 2.1.

В настоящей статье рассматривается в качестве правой части системы (1.1) случайное векторное поле, определенное на произвольном полном вероятностном пространстве $(\Omega, F, P)$,

$$
g=f+\eta
$$

где случайная внешняя сила $f(t, x, \omega)=\left(f_{1}(t, x, \omega), f_{2}(t, x, \omega)\right)^{\mathrm{T}}$ почти наверное является локально квадратично суммируемой по $t$ и случайное векторное поле $\eta(t, x, \omega)=\left(\eta_{1}(t, x, \omega), \eta_{2}(t, x, \omega)\right)^{\mathrm{T}}$ является белым шумом по $t$. Для этой системы доказывается результат о существовании единственного решения задачи Коши со случайными начальными данными и оценка непрерывной зависимости этого решения от совокупности случайных начальных данных и внешней силы на конечном отрезке изменения переменной $t$. Необходимость такой стохастической постановки задачи для изучения проблемы предсказуемости климата подробно описывается в предисловии к книге В.П. Дымникова [7]. Попутно доказывается для задачи Коши с детерминированными начальными данными и правой частью оценка непрерывной зависимости решения от совокупности параметров $\left(\nu, \gamma, \rho, k, k_{1}\right)$, начальных данных и правой части на конечном отрезке изменения переменной $t$.

Автор выражает благодарность В. П. Дымникову и В. Н. Крупчатникову за постановку задачи, А. Р. Ширикяну и В. В. Нерсесяну за полезные дискуссии.

\section{§ 2. Функциональные пространства}

Везде ниже $T>0$ любое, $X$ - банахово пространство с нормой $\|\cdot\|_{X}$.

Пусть $L_{p}(S)$ и $L_{p}([0, T] \times S)$ - лебеговы пространства измеримых функций $\psi: S \rightarrow \mathbb{R}$ и $\psi:[0, T] \times S \rightarrow \mathbb{R}$ соответственно с конечными нормами

$$
\|\psi\|_{L_{p}(S)}=\left(\int_{S}|\psi|^{p} d S\right)^{1 / p}, \quad\|\psi\|_{L_{p}([0, T] \times S)}=\left(\iint_{[0, T] \times S}|\psi|^{p} d t d S\right)^{1 / p}<\infty
$$

$1 \leqslant p<\infty$. При этом $L_{2}(S)$ - гильбертово пространство со скалярным произведением

$$
(\psi, \theta)=\int_{S} \psi \theta d S
$$

Обозначим через $L_{2}(0, T ; X)$ банахово пространство измеримых по Бохнеру функций $\psi:[0, T] \rightarrow X$, для которых конечна норма

$$
\|\psi\|_{L_{2}(0, T ; X)}=\left(\int_{0}^{T}\|\psi(t)\|_{X}^{2} d t\right)^{1 / 2}<\infty
$$


и через $L_{2, \text { lос }}\left(\mathbb{R}^{+} ; X\right)$, где $\mathbb{R}^{+}=\{t \in \mathbb{R}: t \geqslant 0\}$, пространство измеримых по Бохнеру функций $\psi: \mathbb{R}^{+} \rightarrow X$ таких, что

$$
\int_{I}\|\psi(t)\|_{X}^{2} d t<\infty
$$

для любого компактного промежутка $I \subset \mathbb{R}^{+}$. При этом функции, отличающиеся на множествах лебеговой меры нуль, отождествляются. Заметим, что если $X$ - гильбертово пространство, то $L_{2}(0, T ; X)$ - гильбертово пространство.

Нам будут необходимы также пространства непрерывных функций с областью значений в банаховом пространстве. Обозначим через $C(0, T ; X)$ и $C\left(\mathbb{R}^{+} ; X\right)$ пространства всех непрерывных функций $\psi:[0, T] \rightarrow X$ и $\psi: \mathbb{R}^{+} \rightarrow X$ соответственно. Снабженное нормой

$$
\|\psi\|_{C(0, T ; X)}=\max _{t \in[0, T]}\|\psi(t)\|_{X}
$$

пространство $C(0, T ; X)$ является банаховым пространством. Снабженное метрикой

$$
d(\psi, \theta)=\sum_{i=1}^{\infty} 2^{-i} \frac{\|\psi-\theta\|_{C(0, i ; X)}}{1+\|\psi-\theta\|_{C(0, i ; X)}}
$$

пространство $C\left(\mathbb{R}^{+} ; X\right)$ является полным метрическим пространством.

Обозначим через $C^{\infty}(S)$ пространство таких бесконечно гладких функций $\psi: S \rightarrow \mathbb{R}$, для которых выполнено

$$
\int_{S} \psi d S=0
$$

Функция $\psi: S \rightarrow \mathbb{R}$ называется бесконечно гладкой (см., например, замечание 1 к определению 1 в [5; §2] и [8; гл. I, § 3.2]), если для любой декартовой системы координат $\bar{x}=\left(x_{1}, x_{2}, x_{3}\right)^{\mathrm{T}}$ с началом координат в центре единичной сферы $S$ функция

$$
\widetilde{\psi}(\varphi, \lambda)=\psi(\cos \varphi \cos \lambda, \cos \varphi \sin \lambda,-\sin \varphi),
$$

где $\varphi$ и $\lambda$ - углы в сферической системе координат, связанной с декартовой, принадлежит пространству $C^{\infty}((-\pi / 2, \pi / 2) \times[0,2 \pi])$.

Введем в пространстве $C^{\infty}(S)$ семейство норм

$$
\|\psi\|_{p}=\left((-\Delta)^{p} \psi, \psi\right)^{1 / 2}, \quad p \in \mathbb{Z}
$$

Обозначим через $h^{p}, p \in \mathbb{Z}$, пространства, полученные пополнением (см., например, доказательство теоремы о пополнении в $[9 ;$ гл. I, $\S 10])$ по нормам $\|\cdot\|_{p}$, $p \in \mathbb{Z}$, пространства $C^{\infty}(S)$. Имеют место следующие вложения:

$$
\cdots \subset h^{2} \subset h^{1} \subset h^{0} \subset h^{-1} \subset h^{-2} \subset \cdots,
$$

где операторы вложения компактны и непрерывны (см., например, [6; гл. I, §3, леммы $1.1,1.4$, б) и в), 1.6]). Заметим, что $h^{p}$ плотно вложено в $h^{q}$ при $q<p$ (см., например, $[6$; гл. I, §3, леммы 1.1, 1.4, б) и в)]). 
Оператор $-\Delta$ на пространстве $C^{\infty}(S)$ со скалярным произведением $(\cdot, \cdot)$ положительно определен (см., например, [5; $\S 2$, доказательство леммы 1 , формула (22)] и [6; гл. I, §3, лемма 1.2]). Поэтому он может быть расширен до самосопряженного по Фридрихсу (см., например, [10; § 5], [11; §31] и [6; гл. I, §3])

$$
-\Delta: D(-\Delta)=h^{p+2} \subset h^{p} \rightarrow h^{p}, \quad p \in \mathbb{Z},
$$

где $D(A)$ - область определения оператора $A$. Тогда положительно определен и самосопряжен оператор

$$
(-\Delta)^{q / 2}: D\left((-\Delta)^{q / 2}\right)=h^{p+q} \subset h^{p} \rightarrow h^{p}, \quad p, q \in \mathbb{Z}, \quad q>0,
$$

(см., например, [12], [11; §31], [13; гл. ХІІІ] и [6; гл. I, §3]) и

$$
\|\psi\|_{p}=\left((-\Delta)^{p / 2} \psi,(-\Delta)^{p / 2} \psi\right)^{1 / 2} \quad \text { для любого } \psi \in h^{p}, \quad p \in \mathbb{Z} .
$$

Заметим, что скалярное произведение $(\cdot, \cdot)$ в $L_{2}(S)$ может быть продолжено до билинейного непрерывного отображения $h^{p} \times h^{-p} \rightarrow \mathbb{R}, p \in \mathbb{Z}$,

$$
(\psi, \theta)=\left((-\Delta)^{p / 2} \psi,(-\Delta)^{-p / 2} \theta\right), \quad p \in \mathbb{Z},
$$

где $\psi \in h^{p}, \theta \in h^{-p}$. Тогда имеет место обобщенное неравенство Коши-Буняковского

$$
|(\psi, \theta)| \leqslant\|\psi\|_{p}\|\theta\|_{-p} \quad \text { для любых } \psi \in h^{p}, \quad \theta \in h^{-p}, \quad p \in \mathbb{Z},
$$

и (см., например, доказательство [5; § 2, лемма 1] и [6; гл. I, §3], [8; гл. I, § 4.6])

$$
\|\psi\|_{1}^{2}=(-\Delta \psi, \psi)=\||\nabla \psi|\|_{L_{2}(S)}^{2} \quad \text { для любого } \psi \in h^{1},
$$

где в декартовых координатах

$$
\nabla \psi=\left(\frac{\partial}{\partial x_{1}} \psi, \frac{\partial}{\partial x_{2}} \psi, \frac{\partial}{\partial x_{3}} \psi\right)^{\mathrm{T}}, \quad|\theta|=\langle\theta, \theta\rangle_{\mathbb{R}^{n}}^{1 / 2},
$$

$\langle\theta, \xi\rangle_{\mathbb{R}^{n}}$ - скалярное произведение в $\mathbb{R}^{n}, n=2,3$.

Обозначим через $\left\{e_{m}^{n}\right\}_{|m| \leqslant n}^{1 \leqslant n} \in C^{\infty}(S)$ ортонормированный базис гильбертова пространства $h^{0}$ :

$$
\begin{gathered}
\left(e_{m}^{n}, e_{i}^{j}\right)=\delta_{m i} \delta_{n j}, \\
\delta_{m i}, \delta_{n j}-\text { символы Кронекера, } \quad n, j=1,2, \ldots, \quad|m| \leqslant n, \quad|i| \leqslant j,
\end{gathered}
$$

состоящий из собственных функций оператора $-\Delta$ (см., например, [13; гл. XIII] и $[11 ; \S 31])$. Пусть $\lambda_{n}=n(n+1)$ - соответствующее собственным функциям $\left\{e_{m}^{n}\right\}_{|m| \leqslant n}$ собственное значение оператора $-\Delta$ кратности $2 n+1, n=1,2, \ldots$. Отсюда следует, что функции $\left\{e_{m}^{n, p}\right\}_{|m| \leqslant n}^{1 \leqslant n}$, где

$$
e_{m}^{n, p}=\lambda_{n}^{-p / 2} e_{m}^{n}, \quad 1 \leqslant n, \quad|m| \leqslant n,
$$

являются ортонормированным базисом гильбертова пространства $h^{p}, p \in \mathbb{Z} \backslash\{0\}$, если в нем ввести скалярное произведение

$$
(\psi, \theta)_{p}=\left((-\Delta)^{p / 2} \psi,(-\Delta)^{p / 2} \theta\right), \quad p \in \mathbb{Z} \backslash\{0\} .
$$


Пусть $H^{p}=h^{p} \times h^{p}, p \in \mathbb{Z},-$ пространства измеримых векторных полей $\psi: S \rightarrow \mathbb{R}^{2}$ с нормами

$$
\begin{gathered}
\|\psi \mid\|_{p}=\left\langle A_{0}^{p / 2} \psi, A_{0}^{p / 2} \psi\right\rangle^{1 / 2}, \\
p \in \mathbb{Z}, \quad A_{0}=\left(\begin{array}{cc}
-\Delta & 0 \\
0 & -\Delta
\end{array}\right), \quad\langle\psi, \theta\rangle=\int_{S}\langle\psi, \theta\rangle_{\mathbb{R}^{2}} d S .
\end{gathered}
$$

Рассмотрим следующее векторное поле:

$$
E_{m}^{n}= \begin{cases}\left(\begin{array}{c}
0 \\
e_{m-n-1}^{n}
\end{array}\right), & 1 \leqslant m \leqslant 2 n+1, \\
\left(\begin{array}{c}
e_{m-3 n-2}^{n} \\
0
\end{array}\right), & 2 n+2 \leqslant m \leqslant 4 n+2 .\end{cases}
$$

Очевидно, что $\left\{E_{m}^{n}\right\}_{1 \leqslant m \leqslant 4 n+2}^{1 \leqslant n} \in C^{\infty}(S) \times C^{\infty}(S)$ является ортонормированным базисом гильбертова пространства $H^{0}$ :

$$
\left\langle E_{m}^{n}, E_{i}^{j}\right\rangle=\delta_{m i} \delta_{n j}, \quad n, j=1,2, \ldots, \quad 1 \leqslant m \leqslant 4 n+2, \quad 1 \leqslant i \leqslant 4 j+2,
$$

состоящим из собственных векторных полей оператора $A_{0}$. Тогда $\lambda_{n}=n(n+1)$ - соответствующее собственным векторным полям $\left\{E_{m}^{n}\right\}_{1 \leqslant m \leqslant 4 n+2}$ собственное значение оператора $A_{0}$ кратности $4 n+2, n=1,2, \ldots$. Отсюда следует, что векторные поля $\left\{E_{m}^{n, p}\right\}_{1 \leqslant m \leqslant 4 n+2}^{1 \leqslant n}$, где

$$
E_{m}^{n, p}=\lambda_{n}^{-p / 2} E_{m}^{n}, \quad 1 \leqslant n, \quad 1 \leqslant m \leqslant 4 n+2,
$$

являются ортонормированным базисом гильбертова пространства $H^{p}$, где $p \in$ $\mathbb{Z} \backslash\{0\}$, если в нем ввести скалярное произведение

$$
\langle\psi, \theta\rangle_{p}=\left\langle A_{0}^{p / 2} \psi, A_{0}^{p / 2} \theta\right\rangle, \quad p \in \mathbb{Z} \backslash\{0\} .
$$

Введем пространства

$$
\begin{gathered}
\varkappa_{T}=C\left(0, T ; H^{2}\right) \cap L_{2}\left(0, T ; H^{3}\right), \quad\|\psi\|_{\varkappa_{T}}=\|\psi\|_{C\left(0, T ; H^{2}\right)}+\|\psi\|_{L_{2}\left(0, T ; H^{3}\right)}, \\
\varkappa=C\left(\mathbb{R}^{+} ; H^{2}\right) \cap L_{2, \text { loc }}\left(\mathbb{R}^{+} ; H^{3}\right), \\
\Xi_{T}=\left\{\psi \in L_{2}\left(0, T ; H^{3}\right): \frac{\partial}{\partial t} \psi \in L_{2}\left(0, T ; H^{1}\right)\right\},
\end{gathered}
$$

где производная по времени понимается в смысле распределений (см., например, [14; гл. I, § 1.1] или [15; гл. I, § 1.2]). Снабженное нормой

$$
\|\psi\|_{\Xi_{T}}=\left(\int_{0}^{T}\left(\|\| \psi(t) \mid\left\|_{3}^{2}+\right\| \frac{\partial}{\partial t} \psi(t) \|_{1}^{2}\right) d t\right)^{1 / 2}
$$

пространство $\Xi_{T}$ является гильбертовым (доказательство см., например, в [14; гл. I, § 2.2]). Пространство $\Xi_{T}$ непрерывно вложено в пространство $C\left(0, T ; H^{2}\right)$ (доказательство см., например, в [14; гл. I, § 7.3, теорема 7.7, и § 3.1, теорема 3.1] и $\left[6 ;\right.$ гл. I, §3]) и, следовательно, в пространство $\varkappa_{T}$. Поэтому произвольное 
векторное поле $\psi$ из пространства $\Xi_{T}$ является непрерывным как функция $[0, T] \rightarrow H^{2}$ после, быть может, изменения на множестве меры нуль. В частности, при любом $t \in[0, T] \psi(t)$ является векторным полем на пространстве $H^{2}$.

Введем понятие полного вероятностного пространства (см., например, [16; гл. I, $\S 2$ и $\S 4]$ ). Пусть $\Omega$ - произвольное множество, $F$ - некоторое семейство его подмножеств; $F-\sigma$-алгебра, если $\varnothing \in F$ и $F$ замкнуто относительно операций дополнения и объединения счетного числа множеств из $F$. Пару $(\Omega, F)$ называют измеримым пространством, если $F-\sigma$-алгебра подмножеств $\Omega$. $M e$ рой называют неотрицательную счетно-аддитивную функцию, определенную на некоторой $\sigma$-алгебре измеримого пространства; $P$ - вероятностная мера на измеримом пространстве $(\Omega, F)$, если $P(\Omega)=1$. Тройку $(\Omega, F, P)$ называют вероятностным пространством. Под пополнением $\mathrm{F}_{P} \sigma$-алгебры $F$ по вероятностной мере $P$ будем понимать $F \cup N_{P}$, где $N_{P}$ - семейство подмножеств $M \subset \Omega$ таких, что существует подмножество $N \subset \Omega, N \in F$, что $M \subset N$ и $P(N)=0$. Если $\mathrm{F}_{P}=F$, то $(\Omega, F, P)$ - полное вероятностное пространство.

В дальнейшем полагаем, что $(\Omega, F, P)$ - полное вероятностное пространство.

Определим банахово пространство $L_{2}(\Omega ; X)$ (см., например, [17; гл. II, $\S 10.5$, теорема 7]) измеримых по Бохнеру функций $\psi: \Omega \rightarrow X$ таких, что конечна норма

$$
\|\psi\|_{L_{2}(\Omega ; X)}=\sqrt{E\|\psi\|_{X}^{2}}=\left(\int_{\Omega}\|\psi\|_{X}^{2} d P\right)^{1 / 2}<\infty .
$$

При этом функции, отличающиеся на множествах $P$-меры нуль, отождествляются.

В следующих двух абзацах приведены определения из [18; гл. II, § 1, и гл. IV, $\S 5]$ и $[19 ;$ гл. I, § 1.1 и $\S 3.3]$.

В пространстве $(\Omega, F, P)$ введем фильтрацию $\left\{F_{t}\right\}_{t \geqslant 0}-$ неубывающее семейство $\sigma$-алгебр $F_{s} \subset F_{t}, s<t$, при этом $F_{t} \subset F, t \geqslant 0$. Фильтрацию $\left\{F_{t}\right\}_{t \geqslant 0}$ назовем непрерывной справа, если

$$
\bigcap_{s>t} F_{s}=F_{t}, \quad t \geqslant 0 .
$$

Будем говорить, что фильтрация $\left\{F_{t}\right\}_{t \geqslant 0}$ удовлетворяет обычным условиям, если $\left\{F_{t}\right\}_{t \geqslant 0}-$ непрерывная справа фильрация такая, что все множества $P$-меры нуль $\sigma$-алгебры $F$ содержатся в $F_{0}$.

В дальнейшем полагаем, что фильтрация $\left\{F_{t}\right\}_{t \geqslant 0}$ удовлетворяет обычным условиям.

Наименьшую $\sigma$-алгебру, содержащую все открытые множества из $X$, называют борелевской $\sigma$-алгеброй $X$. Обозначим через $\sigma_{X}$ борелевскую $\sigma$-алгебру $X$. Отображение $\psi:(\Omega, F) \rightarrow\left(X, \sigma_{X}\right)$ называют $F \mid \sigma_{X}$-измеримым, если $\{\omega \in \Omega$ : $\psi(\omega) \in M\} \in F$ для любого $M \in \sigma_{X}$. Случайный процесс $\xi(t): \Omega \rightarrow X, t \geqslant 0$, согласован с фильтращией $\left\{F_{t}\right\}_{t \geqslant 0}$, если отображение $\xi(t) F_{t} \mid \sigma_{X}$-измеримо при каждом $t \geqslant 0$. Случайный процесс $\xi(t): \Omega \rightarrow X, t \geqslant 0$, прогрессивно измерим по отношению $\kappa\left\{F_{t}\right\}_{t \geqslant 0}$, если для любого $t \geqslant 0$ отображение $(s, \omega) \rightarrow \xi(s, \omega)$, где $(s, \omega) \in[0, t] \times \Omega$, является $\sigma_{[0, t]} \times F_{t} \mid \sigma_{X}$-измеримым. Здесь $\sigma_{[0, t]} \times F_{t}-$ наименьшая $\sigma$-алгебра подмножеств $[0, t] \times \Omega$, содержащая все множества $M_{1} \times M_{2}$, где $M_{1} \in \sigma_{[0, t]}$ и $M_{2} \in F_{t}$. 
Введем гильбертово пространство $l_{2}$ последовательностей $\left\{b_{i}\right\}_{i=1}^{\infty}$ действительных чисел с конечной нормой

$$
\left\|\left\{b_{i}\right\}_{i=1}^{\infty}\right\|_{l_{2}}=\left(\sum_{i=1}^{\infty} b_{i}^{2}\right)^{1 / 2}<\infty
$$

и подмножество этого пространства

$$
l_{2}^{+}=\left\{\left\{b_{i}\right\}_{i=1}^{\infty} \in l_{2}: b_{i} \geqslant 0, i=1,2, \ldots\right\} .
$$

\section{§ 3. Теорема о существовании единственного решения задачи Коши для системы уравнений (1.1), (1.2)}

Пусть заданы случайное векторное поле $f(t, x, \omega), t \geqslant 0$, согласованное с фильтрацией $\left\{F_{t}\right\}_{t \geqslant 0}, f \in L_{2, \text { loc }}\left(\mathbb{R}^{+} ; H^{-1}\right)$ почти наверное, и $F_{0}$-измеримое случайное векторное поле $u_{0}(x, \omega), u_{0} \in H^{2}$ почти наверное. Рассмотрим задачу Коши для системы (1.1), (1.2)

$$
u(0)=u_{0} .
$$

Обозначим через $\left\{E_{i}\right\}_{i=1}^{\infty}$ ортонормированный базис пространства $H^{0}$. В качестве случайного векторного поля рассмотрим

$$
\eta(t, x, \omega)=\frac{\partial}{\partial t} \zeta(t, x, \omega), \quad \zeta(t, x, \omega)=\sum_{i=1}^{\infty} b_{i} \beta_{i}(t, \omega) E_{i},
$$

где $\left\{b_{i}\right\}_{i=1}^{\infty} \in l_{2}^{+},\left\{\beta_{i}(t, \omega)\right\}_{i=1}^{\infty}, t \geqslant 0,-$ последовательность независимых действительных броуновских движений, определенных на полном вероятностном пространстве $(\Omega, F, P)$, относительно фильтрации $\left\{F_{t}\right\}_{t \geqslant 0}$ (см. определение 7 в [16; “Дополнения и упражнения" к гл. III]). Тогда ряд в (3.2) принадлежит пространству $C\left(\mathbb{R}^{+} ; H^{0}\right)$ для почти всех $\omega \in \Omega$ (см., например, доказательство [19; гл. I, §4.1, теорема 4.3]).

Поскольку почти все траектории броуновского движения не дифференцируемы ни в одной точке (см., например, [16; гл. III, §1, теорема 1]) и производная броуновского движения существует почти наверное только в обобщенном смысле (см., например, [20; гл. III, § 1.4]), то необходимо придать смысл системе $(1.1),(1.2)$. Будем считать, что это формальная запись стохастического интегрального уравнения. То есть введем следующее определение решения системы (1.1), (1.2).

ОПредЕлЕНИЕ 1 . Случайный процесс $u(t, x, \omega), t \geqslant 0$, определенный на вероятностном пространстве $(\Omega, F, P)$, со значениями в $H^{2}$ будем называть решением системы (1.1), (1.2), если он обладает следующими свойствами:

a) случайный процесс $u(t), t \geqslant 0$, согласован с фильтрацией $\left\{F_{t}\right\}_{t \geqslant 0}$ и почти все его траектории принадлежат пространству $\varkappa$;

b) почти все траектории случайного процесса $u(t)$ удовлетворяют равенству $A_{1} u(t)+\int_{0}^{t}\left(\nu A_{2} u(s)+A_{3} u(s)+B(u(s))\right) d s=A_{1} u(0)+\int_{0}^{t} f(s) d s+\zeta(t), \quad t>0$, которое имеет место в пространстве $C\left(\mathbb{R}^{+} ; H^{-1}\right)$. 
Для построения решения задачи Коши (1.1), (1.2), (3.1) рассмотрим еще одну стохастическую систему

$$
\frac{\partial}{\partial t} A_{1} z+\nu A_{2} z+A_{3} z=\eta, \quad t>0
$$

где $z(t, x, \omega)=\left(z_{1}(t, x, \omega), z_{2}(t, x, \omega)\right)^{\mathrm{T}}$. Решение этой системы определяется аналогично определению решения системы $(1.1),(1.2)$. При этом вместо (3.3) будем иметь

$$
A_{1} z(t)+\int_{0}^{t}\left(\nu A_{2} z(s)+A_{3} z(s)\right) d s=A_{1} z(0)+\zeta(t), \quad t>0 .
$$

Имеет место следующее

ПРеДЛОЖЕНИЕ 1. Для любъх $\nu>0, \gamma, \rho, k, k_{1} \geqslant 0,\left\{b_{i}\right\}_{i=1}^{\infty} \in l_{2}^{+}$cуществует решение $z(t)$ системы (3.4) с начальным условием $z(0)=0$, и оно единственно, т.е. если $\widetilde{z}(t)$ - другое решение системы (3.4) с начальным условием $\widetilde{z}(0)=0$, то $z(t)=\widetilde{z}(t)$ для любого $t \geqslant 0$ с вероятностью 1 .

Доказательство этого предложения будет приведено ниже.

Поскольку $f \in L_{2, \text { loc }}\left(\mathbb{R}^{+} ; H^{-1}\right)$ почти наверное, $u_{0} \in H^{2}$ почти наверное и по предложению 1 случайное векторное поле $z \in \varkappa$ почти наверное, то рассмотрим множество

$$
\Omega_{*}=\left\{\omega \in \Omega: f \in L_{2, \mathrm{loc}}\left(\mathbb{R}^{+} ; H^{-1}\right), u_{0} \in H^{2}, z \in \varkappa\right\}
$$

полной меры $P\left(\Omega_{*}\right)=1$. Такое множество существует в силу свойства множеств полной меры: конечное пересечение множеств полной меры является множеством полной меры.

Будем искать решение задачи Коши (1.1), (1.2), (3.1) в виде суммы $u(t)=$ $v(t)+z(t)$, где $z(t)$ - решение системы (3.4) с начальным условием $z(0)=0$. Тогда случайный процесс $v(t)$ удовлетворяет задаче Коши

$$
\frac{\partial}{\partial t} A_{1} v+\nu A_{2} v+A_{3} v+B(v+z)=f, \quad t>0, \quad v(0)=u_{0} .
$$

В задаче Коши (3.7) зафиксируем произвольное $\omega \in \Omega_{*}$ и в дальнейшем для удобства изложения будем считать эту задачу Коши детерминированной до изложения основного результата, доказанного в теореме 2 в конце этого параграфа.

ОПРЕДЕЛЕниЕ 2. Пусть $z(t, x)$ - векторное поле из пространства $\varkappa_{T}$. Векторное поле $v(t, x)$ будем называть решением задачи Коши $(3.7)$ на $[0, T] \times S$, если $v \in \Xi_{T}, v(0)=u_{0}$ и уравнение в (3.7) имеет место в $L_{2}\left(0, T ; H^{-1}\right)$.

Для построения решения задачи Коши (3.7) нам будут необходимы несколько вспомогательных результатов.

Лемма 1. І. Пусть $\theta, \xi \in h^{2}, \chi \in h^{0}$. Имеет место оценка

$$
|(J(\theta, \xi), \chi)| \leqslant C\left(\|\theta\|_{2}\|\theta\|_{1}\|\xi\|_{2}\|\xi\|_{1}\right)^{1 / 2}\|\chi\|_{0} .
$$

II. Пусть $\xi, \chi \in h^{1}, \theta \in h^{2}$. Имеет место оценка

$$
|(J(\theta, \xi), \chi)| \leqslant C\left(\|\theta\|_{2}\|\theta\|_{1}\|\chi\|_{1}\|\chi\|_{0}\right)^{1 / 2}\|\xi\|_{1} .
$$


III. Пусть $\theta \in h^{1}, \xi \in h^{0}$. Имеет место оценка

$$
|(J(\mu, \theta), \xi)| \leqslant\|\theta\|_{1}\|\xi\|_{0} .
$$

Константа $C>0$ не зависит от $\theta, \xi, \chi$.

ДоказАтельство. І. Разложив $\psi$ по базису $\left\{e_{m}^{n, 2}\right\}_{|m| \leqslant n}^{1 \leqslant n}$ пространства $h^{2}$, легко доказать неравенство

$$
\left((-\Delta)^{3 / 2} \psi, \psi\right) \leqslant\|\psi\|_{2}\|\psi\|_{1} \quad \text { для любого } \psi \in h^{2} .
$$

Поэтому, используя лемму 2 с параметрами $p=2, q=4, r=3 / 2, s=1$ и лемму 3 при $p=4$ из статьи [21], нетрудно получить оценку

$$
\||\nabla \psi|\|_{L^{4}(S)} \leqslant C_{1}\|\psi\|_{2}^{1 / 2}\|\psi\|_{1}^{1 / 2} \quad \text { для любого } \psi \in h^{2},
$$

где константа $C_{1}>0$ не зависит от $\psi$. Тогда в силу того, что произведение двух функций с интегрируемым квадратом есть интегрируемая функция (см., например, [22; гл. VII, §2.1, свойство 1]), интегрируема функция $|\nabla \theta|^{2}|\nabla \xi|^{2}$. По неравенству Коши-Буняковского для пространства $L_{2}(S)$ имеем

$$
\||\nabla \theta||\nabla \xi|\|_{L_{2}(S)} \leqslant\||\nabla \theta|\|_{L_{4}(S)}\||\nabla \xi|\|_{L_{4}(S)} .
$$

Рассмотрим

$$
\begin{aligned}
\nabla \psi & =\left(0, \sqrt{1-\mu^{2}} \frac{\partial}{\partial \mu} \psi, \frac{1}{\sqrt{1-\mu^{2}}} \frac{\partial}{\partial \lambda} \psi\right)^{\mathrm{T}}, \\
\nabla^{\perp} \psi & =\left(0, \frac{1}{\sqrt{1-\mu^{2}}} \frac{\partial}{\partial \lambda} \psi,-\sqrt{1-\mu^{2}} \frac{\partial}{\partial \mu} \psi\right)^{\mathrm{T}},
\end{aligned}
$$

где вектор $\nabla \psi$ записан в сферической системе координат (см., например, [5; $\S 1])$. В силу неравенства Коши-Буняковского для $\mathbb{R}^{3}$ почти всюду

$$
|J(\theta, \xi)|=\left|\left\langle\nabla^{\perp} \theta, \nabla \xi\right\rangle_{\mathbb{R}^{3}}\right| \leqslant\left|\nabla^{\perp} \theta\right||\nabla \xi|=|\nabla \theta||\nabla \xi| .
$$

Поэтому в силу свойств интеграла Лебега (см., например, $[22 ;$ гл. V, § 5.3, свойство 7 и неравенство (12)]) интегрируема функция $|J(\theta, \xi)|^{2}$ и

$$
\|J(\theta, \xi)\|_{L_{2}(S)} \leqslant\||\nabla \theta||\nabla \xi|\|_{L_{2}(S)} \leqslant\||\nabla \theta|\|_{L_{4}(S)}\|\mid \nabla \xi\|_{L_{4}(S)} .
$$

Тогда ввиду свойств функций из $L_{2}(S)$ (см., например, [22; гл. VII, $\S 2.1$, свойство 1]) интегрируема функция $J(\theta, \xi) \chi$ и по неравенству Коши-Буняковского для пространства $L_{2}(S)$ имеем

$$
|(J(\theta, \xi), \chi)|=\left|\int_{S} J(\theta, \xi) \chi d S\right| \leqslant\|J(\theta, \xi)\|_{L_{2}(S)}\|\chi\|_{L_{2}(S)}=\|J(\theta, \xi)\|_{L_{2}(S)}\|\chi\|_{0} .
$$

Отсюда и из (3.8) и (3.10) сразу следует первое неравенство в лемме.

II. Доказательство аналогично. Необходимо применить по-другому неравенство Коши-Буняковского для пространства $L_{2}(S)$ к $|(J(\theta, \xi), \chi)|$ :

$$
|(J(\theta, \xi), \chi)| \leqslant\||\nabla \theta|\|_{L_{4}(S)}\|\chi\|_{L_{4}(S)}\||\nabla \xi|\|_{L_{2}(S)},
$$


и помимо оценки (3.8) использовать аналогичную оценку (доказательство см., например, в $[23 ; \S 7$, лемма 9])

$$
\|\psi\|_{L_{4}(S)} \leqslant C_{2}\|\psi\|_{1}^{1 / 2}\|\psi\|_{0}^{1 / 2} \text { для любого } \psi \in h^{1},
$$

где константа $C_{2}>0$ не зависит от $\psi$.

III. Поскольку $\mu \in C^{\infty}(S), J(\mu, \theta)=\theta_{\lambda}, 0 \leqslant 1-\mu^{2} \leqslant 1$, то, учитывая (2.3), $(3.9)$, из свойств функций из $L_{2}(S)$ (см., например, [22; гл. VII, $\S 2.1$, свойство 1]) функция $J(\mu, \theta) \xi$ интегрируема. Неравенство в лемме сразу следует из неравенства Коши-Буняковского для пространства $L_{2}(S)$ и из $(2.3)$.

Лемма доказана.

Нетрудно доказать следующую лемму, которая была сформулирована в работе [24; гл. II, §1.3, лемма 2.1.4].

Лемма 2. Пусть каждое векторное пространство $V_{i}$ плотно в банаховом пространстве $X_{i}$ при $i=1,2, \ldots, j, Y$ - банахово пространство, $j$-линейное отображение $F: V_{1} \times V_{2} \times \cdots \times V_{j} \rightarrow Y$ таково, что для любых $v_{1} \in V_{1}, v_{2} \in V_{2}$, $\ldots, v_{j} \in V_{j}$ выполнено неравенство

$$
\left\|F\left(v_{1}, v_{2}, \ldots, v_{j}\right)\right\|_{Y} \leqslant C\left\|v_{1}\right\|_{X_{1}}\left\|v_{2}\right\|_{X_{2}} \cdots\left\|v_{j}\right\|_{X_{j}}
$$

где $C>0$ - константа, не зависящая от $v_{i}, i=1,2, \ldots, j,\|\cdot\|$ - норма в соответствующих пространствах. Тогда отображсение $F$ может быть продолжено единственным образом до непрерывного на пространство $X_{1} \times$ $X_{2} \times \cdots \times X_{j} u(3.12)$ будет выполнено для любъх $v_{1} \in X_{1}, v_{2} \in X_{2}, \ldots$, $v_{j} \in X_{j}$.

ЛЕмма 3. Пусть выполнено одно из условий:

$$
\begin{aligned}
& \theta, \xi \in h^{2}, \quad \chi \in h^{0} ; \quad \chi, \theta \in h^{2}, \quad \xi \in h^{0} ; \quad \xi, \chi \in h^{2}, \quad \theta \in h^{0} ; \\
& \theta, \xi \in h^{1}, \quad \chi \in h^{2} ; \quad \chi, \theta \in h^{1}, \quad \xi \in h^{2} ; \quad \xi, \chi \in h^{1}, \quad \theta \in h^{2} \text {. }
\end{aligned}
$$

Тогда якобиан J обладает следующими свойствами:

$$
\begin{gathered}
(J(\theta, \xi), \chi)=-(J(\xi, \theta), \chi) \\
(J(\theta, \xi), \chi)=(J(\chi, \theta), \xi)=(J(\xi, \chi), \theta) .
\end{gathered}
$$

Eсли $\theta \in h^{2}, \xi \in h^{0}$ uлu $\theta \in h^{1}, \xi \in h^{2}$, mo

$$
(J(\theta, \xi), \theta)=0 .
$$

Eсли $\theta \in h^{2}$, mo

$$
(J(\theta, \mu), \Delta \theta)=0 .
$$

ДокАзАтЕльство. По лемме 2.1, формулам (2.2.8), (2.2.10) из [6; гл. II, §2] эти равенства справедливы для функций из класса $C^{\infty}(S)$. Из непрерывности вложений $h^{2}$ в $h^{1}$ и $h^{1}$ в $h^{0}$, плотности $C^{\infty}(S)$ в $h^{0}, h^{1}$ и $h^{2}$, леммы 1 , леммы 2 и того, что $\mu \in C^{\infty}(S)$, сразу следуют равенства для $\theta, \xi, \chi$ из тех функциональных пространств, которые указаны в условии леммы.

Лемма доказана. 
Лемма 4. Пусть $\theta \in C\left(0, T ; h^{0}\right) \cap L_{2}\left(0, T ; h^{1}\right), \xi \in C\left(0, T ; h^{1}\right) \cap L_{2}\left(0, T ; h^{2}\right)$. Тогда имеет место оценка

$$
\|\theta|\nabla \xi|\|_{L_{2}([0, T] \times S)} \leqslant C\left(\|\theta\|_{C\left(0, T ; h^{0}\right)}\|\theta\|_{L_{2}\left(0, T ; h^{1}\right)}\|\xi\|_{C\left(0, T ; h^{1}\right)}\|\xi\|_{L_{2}\left(0, T ; h^{2}\right)}\right)^{1 / 2}
$$

где константа $C>0$ не зависит от $\theta$ и $\xi$.

ДокАЗАТЕльство. В силу (3.8), (3.11) выполнены неравенства для почти Bcex $t \in[0, T]$

$$
\begin{gathered}
\|\theta(t)\|_{L_{4}(S)}^{4} \leqslant C\|\theta\|_{C\left(0, T ; h^{0}\right)}^{2}\|\theta(t)\|_{h^{1}}^{2}, \\
\|\nabla \xi(t)\|\left\|_{L_{4}(S)}^{4} \leqslant C\right\| \xi\left\|_{C\left(0, T ; h^{1}\right)}^{2}\right\| \xi(t) \|_{h^{2}}^{2},
\end{gathered}
$$

где константа $C>0$ не зависит от $\theta$ и $\xi$. Ввиду свойств интеграла Лебега (см., например, [22; гл. $\mathrm{V}, \S 5.3$, свойство 7]) интегрируемы функции $\|\theta(t)\|_{L_{4}(S)}^{4}$ и $\||\nabla \xi(t)|\|_{L_{4}(S)}^{4}$. По теореме Фубини (см., например, [22; гл. V, §6.4, замечание])

$$
\begin{gathered}
\int_{0}^{T}\|\theta(t)\|_{L_{4}(S)}^{4} d t=\|\theta\|_{L_{4}([0, T] \times S)}^{4}<\infty, \\
\int_{0}^{T}\||\nabla \xi(t)|\|_{L_{4}(S)}^{4} d t=\|\mid \nabla \xi\|_{L_{4}([0, T] \times S)}^{4}<\infty .
\end{gathered}
$$

Поэтому

$$
\begin{gathered}
\|\theta\|_{L_{4}([0, T] \times S)} \leqslant C\|\theta\|_{C\left(0, T ; h^{0}\right)}^{1 / 2}\|\theta\|_{L_{2}\left(0, T ; h^{1}\right)}^{1 / 2}, \\
\|\mid \nabla \xi\|_{L_{4}([0, T] \times S)} \leqslant C\|\xi\|_{C\left(0, T ; h^{1}\right)}^{1 / 2}\|\xi\|_{L_{2}\left(0, T ; h^{2}\right)}^{1 / 2}
\end{gathered}
$$

Ввиду свойств функций из $L_{2}([0, T] \times S)$ (см., например, [22; гл. VII, $\S 2.1$, свойство 1]) интегрируема функция $\theta^{2}|\nabla \xi|^{2}$, и по неравенству Коши-Буняковского для пространства $L_{2}([0, T] \times S)$

$$
\|\theta|\nabla \xi|\|_{L_{2}([0, T] \times S)} \leqslant\|\theta\|_{L_{4}([0, T] \times S)}\||\nabla \xi|\|_{L_{4}([0, T] \times S)} .
$$

Отсюда сразу вытекает требуемое неравенство.

Лемма доказана.

Введем одно определение.

ОПРЕДЕЛЕНИЕ 3. Пусть $M_{i}$ - подмножество, содержащее нулевой элемент, в нормированном пространстве $X_{i}, i=1,2, \ldots, j, Y$ - нормированное пространство. Отображение

$$
\Phi: \mathbb{R}^{+} \backslash\{0\} \times M_{1} \times M_{2} \times \cdots \times M_{j} \rightarrow Y
$$

будем называть равномерно непрерывным по Липшицу на ограниченных подмножествах, если для любых $\mathrm{R}_{0}>0, \mathrm{R}_{1}, \mathrm{R}_{2}, \ldots, \mathrm{R}_{j} \geqslant 0$ и любого $r_{0}>0$, $r_{0} \leqslant \mathrm{R}_{0}$, существует константа $C=C\left(r_{0}, \mathrm{R}_{0}, \mathrm{R}_{1}, \ldots, \mathrm{R}_{j}\right) \geqslant 0$ такая, что имеет место неравенство

$$
\left\|\Phi\left(x_{0}^{\mathrm{I}}, x_{1}^{\mathrm{I}}, \ldots, x_{j}^{\mathrm{I}}\right)-\Phi\left(x_{0}^{\mathrm{II}}, x_{1}^{\mathrm{II}}, \ldots, x_{j}^{\mathrm{II}}\right)\right\|_{Y} \leqslant C\left(\left|x_{0}^{\mathrm{I}}-x_{0}^{\mathrm{II}}\right|+\sum_{i=1}^{j}\left\|x_{i}^{\mathrm{I}}-x_{i}^{\mathrm{II}}\right\|_{X_{i}}\right),
$$


где $x_{0}^{\mathrm{I}}, x_{0}^{\mathrm{II}}$ - произвольные вещественные числа из отрезка $\left[r_{0}, \mathrm{R}_{0}\right], x_{i}^{\mathrm{I}}, x_{i}^{\mathrm{II}} \in M_{i}$, $\left\|x_{i}^{\mathrm{I}}\right\|_{X_{i}},\left\|x_{i}^{\mathrm{II}}\right\|_{X_{i}} \leqslant \mathrm{R}_{i}, i=1,2, \ldots, j,-$ произвольные элементы соответствующих подмножеств, константа $C$ не зависит от $\left(x_{0}^{\mathrm{I}}, x_{1}^{\mathrm{I}}, \ldots, x_{j}^{\mathrm{I}}\right)$ и $\left(x_{0}^{\mathrm{II}}, x_{1}^{\mathrm{II}}, \ldots, x_{j}^{\mathrm{II}}\right)$.

Следующий результат о свойствах решений задачи Коши (3.7) является ключевым для построения решения задачи Коши (1.1), (1.2), (3.1).

Теорема 1. Пусть заданъ векторные поля $f \in L_{2}\left(0, T ; H^{-1}\right), z \in \varkappa_{T}$ при некотором $T>0, u_{0} \in H^{2}$. Тогда существует единственное решение задачи Коши (3.7) на $[0, T] \times S$ для любых $\nu>0, \gamma, \rho, k, k_{1} \geqslant 0$. Кроме того, оператор

$$
\Phi: \mathbb{R}^{+} \backslash\{0\} \times\left(\mathbb{R}^{+}\right)^{4} \times L_{2}\left(0, T ; H^{-1}\right) \times H^{2} \times \varkappa_{T} \rightarrow \Xi_{T} \subset \varkappa_{T},
$$

который каждому вектору $\left(\nu, \gamma, \rho, k, k_{1}, f, u_{0}, z\right)$ сопоставляет решение $v$ задачи Коши (3.7) на $[0, T] \times S$ является равномерно непрерывным по Липшичу на ограниченных подмножествах, если его рассматривать как действующий в $\Xi_{T}$ и как действующий в $\varkappa_{T}$.

ДокАЗАТЕЛЬство. Единственность. Пусть существуют два решения $v^{\mathrm{I}}(t)$ и $v^{\mathrm{II}}(t)$ задачи Коши $(3.7)$ на $[0, T] \times S$. Тогда разность $w(t)=v^{\mathrm{I}}(t)-v^{\mathrm{II}}(t)$ удовлетворяет следующей задаче Коши:

$$
\frac{\partial}{\partial t} A_{1} w+\nu A_{2} w+A_{3} w+B\left(v^{\mathrm{I}}+z\right)-B\left(v^{\mathrm{II}}+z\right)=0, \quad t>0, \quad w(0)=0 .
$$

Умножая скалярно уравнение в (3.14) на $w$, получаем в силу $(2.1)$

$$
\left\langle\frac{\partial}{\partial t} A_{1} w, w\right\rangle+\nu\left\langle A_{2} w, w\right\rangle+\left\langle A_{3} w, w\right\rangle+\left\langle B\left(v^{\mathrm{I}}+z\right)-B\left(v^{\mathrm{II}}+z\right), w\right\rangle=0 .
$$

Отсюда, учитывая, что аналогично доказательству леммы 1.2 из [25; гл. III, $\S 1.4]$ нетрудно получить равенство

$$
\left\langle\frac{\partial}{\partial t} A_{1} w, w\right\rangle=\frac{1}{2} \frac{\partial}{\partial t}\left\langle A_{1} w, w\right\rangle
$$

имеем в силу $(2.1)$

$$
\frac{1}{2} \frac{\partial}{\partial t}\left\langle A_{1} w, w\right\rangle+\nu\left|\|w \mid\|_{2}^{2}+\left\langle A_{3} w, w\right\rangle=\left\langle B\left(v^{\mathrm{II}}+z\right)-B\left(v^{\mathrm{I}}+z\right), w\right\rangle .\right.
$$

Оценим снизу $\left\langle A_{3} \psi, \psi\right\rangle, \psi \in H^{1}$. По определению оператора $A_{3}$, используя $(2.1),(2.2)$ и учитывая, что $\nu>0, \gamma, \rho, k, k_{1} \geqslant 0$, получим

$$
\begin{aligned}
\left\langle A_{3} \psi, \psi\right\rangle & =\left\langle\left(\begin{array}{c}
-k \Delta \psi_{1}+2 k \Delta \psi_{2} \\
k \Delta \psi_{1}-\left(2 k+k_{1}+\nu \gamma\right) \Delta \psi_{2}+\rho \psi_{2}
\end{array}\right), \psi\right\rangle \\
& =k\left\|\psi_{1}\right\|_{1}^{2}+2 k\left(\Delta \psi_{2}, \psi_{1}\right)+k\left(\Delta \psi_{1}, \psi_{2}\right)+\left(2 k+k_{1}+\nu \gamma\right)\left\|\psi_{2}\right\|_{1}^{2}+\rho\left\|\psi_{2}\right\|_{0}^{2} \\
& \geqslant k\left\|\psi_{1}\right\|_{1}^{2}-3 k\left\|\psi_{1}\right\|_{1}\left\|\psi_{2}\right\|_{1}+2 k\left\|\psi_{2}\right\|_{1}^{2} .
\end{aligned}
$$

Из неравенств $k \geqslant 0$ и

$$
a^{2}-3 a b+3 b^{2} \geqslant 0, \quad a, b \in \mathbb{R},
$$

имеем

$$
\left\langle A_{3} \psi, \psi\right\rangle \geqslant-k\left\|\psi_{2}\right\|_{1}^{2} \geqslant-k\|\| \psi \mid \|_{1}^{2} \quad \text { для любого } \psi \in H^{1} .
$$


Оценим сверху $\left|\left\langle B\left(v^{\mathrm{I}}+z\right)-B\left(v^{\mathrm{II}}+z\right), w\right\rangle\right|$. По определению $B(u)$

$$
\begin{aligned}
\left|\left\langle B\left(v^{\mathrm{I}}+z\right)-B\left(v^{\mathrm{II}}+z\right), w\right\rangle\right| & \\
=\mid & \left\langle\left(\begin{array}{c}
J\left(\Delta\left(v_{1}^{\mathrm{I}}+z_{1}\right)+2 \mu, w_{1}\right)+J\left(\Delta\left(v_{2}^{\mathrm{I}}+z_{2}\right), w_{2}\right) \\
J\left(\Delta\left(v_{2}^{\mathrm{I}}+z_{2}\right)-\gamma\left(v_{2}^{\mathrm{I}}+z_{2}\right), w_{1}\right)+J\left(\Delta\left(v_{1}^{\mathrm{I}}+z_{1}\right)+2 \mu, w_{2}\right)
\end{array}\right)\right. \\
& \left.\left.\quad-\left(\begin{array}{c}
J\left(\Delta w_{1}, v_{1}^{\mathrm{II}}+z_{1}\right)+J\left(\Delta w_{2}, v_{2}^{\mathrm{II}}+z_{2}\right) \\
J\left(\Delta w_{2}-\gamma w_{2}, v_{1}^{\mathrm{II}}+z_{1}\right)+J\left(\Delta w_{1}, v_{2}^{\mathrm{II}}+z_{2}\right)
\end{array}\right), w\right\rangle\right) \mid \\
=\mid & \left(J\left(\Delta\left(v_{1}^{\mathrm{I}}+z_{1}\right)+2 \mu, w_{1}\right), w_{1}\right)+\left(J\left(\Delta\left(v_{2}^{\mathrm{I}}+z_{2}\right), w_{2}\right), w_{1}\right) \\
& +\left(J\left(\Delta\left(v_{2}^{\mathrm{I}}+z_{2}\right)-\gamma\left(v_{2}^{\mathrm{I}}+z_{2}\right), w_{1}\right), w_{2}\right)+\left(J\left(\Delta\left(v_{1}^{\mathrm{I}}+z_{1}\right)+2 \mu, w_{2}\right), w_{2}\right) \\
& -\left(J\left(\Delta w_{1}, v_{1}^{\mathrm{II}}+z_{1}\right), w_{1}\right)-\left(J\left(\Delta w_{2}, v_{2}^{\mathrm{II}}+z_{2}\right), w_{1}\right) \\
& -\left(J\left(\Delta w_{2}-\gamma w_{2}, v_{1}^{\mathrm{II}}+z_{1}\right), w_{2}\right)-\left(J\left(\Delta w_{1}, v_{2}^{\mathrm{II}}+z_{2}\right), w_{2}\right) \mid .
\end{aligned}
$$

В силу леммы 3 и того, что $\mu \in C^{\infty}(S)$,

$$
\begin{aligned}
\left|\left\langle B\left(v^{\mathrm{I}}+z\right)-B\left(v^{\mathrm{II}}+z\right), w\right\rangle\right| & \\
=\mid & \gamma\left(J\left(v_{2}^{\mathrm{I}}+z_{2}, w_{1}\right), w_{2}\right)+\left(J\left(\Delta w_{1}, v_{1}^{\mathrm{II}}+z_{1}\right), w_{1}\right)+\left(J\left(\Delta w_{2}, v_{2}^{\mathrm{II}}+z_{2}\right), w_{1}\right) \\
& +\left(J\left(\Delta w_{2}, v_{1}^{\mathrm{II}}+z_{1}\right), w_{2}\right)+\left(J\left(\Delta w_{1}, v_{2}^{\mathrm{II}}+z_{2}\right), w_{2}\right) \mid .
\end{aligned}
$$

Тогда по лемме 1, п. I, равенству (3.13) и из непрерывности вложения пространства $h^{2}$ в $h^{0}$ получим

$$
\left|\left\langle B\left(v^{\mathrm{I}}+z\right)-B\left(v^{\mathrm{II}}+z\right), w\right\rangle\right| \leqslant C_{1} \max _{i=\mathrm{I}, \mathrm{II}}\left(\left|\left\|v^{i}+z\left|\left\|_ { 2 } | \| v ^ { i } + z | \| _ { 1 } ) ^ { 1 / 2 } \left|\left\|w \left|\left\|_ { 2 } ^ { 3 / 2 } \left|\|w \mid\|_{1}^{1 / 2},\right.\right.\right.\right.\right.\right.\right.\right.\right.\right.
$$

где константа $C_{1}>0$ не зависит от $v^{\mathrm{I}}$ и $v^{\mathrm{II}}$. Отсюда, используя очевидное неравенство

$$
a^{3} b \leqslant a^{3} b+b^{3} a \leqslant a^{4}+b^{4}, \quad a, b>0,
$$

выводим оценку

$$
\left|\left\langle B\left(v^{\mathrm{I}}+z\right)-B\left(v^{\mathrm{II}}+z\right), w\right\rangle\right| \leqslant \nu|||w|\left\|_{2}^{2}+C_{2} \max _{i=\mathrm{I}, \mathrm{II}}\left(\left|\left\|v^{i}+z\left|\left\|_{2}\left|\left\|v^{i}+z \mid\right\|_{1}\right)^{2}|||w|\right\|_{1}^{2},\right.\right.\right.\right.\right.
$$

где $C_{2}=C_{1}^{4} / \nu^{3}$.

Из (3.15)-(3.17) вытекает оценка

$\frac{1}{2} \frac{\partial}{\partial t}\left\langle A_{1} w, w\right\rangle+\nu\left|\left\|w\left|\left\|_{2}^{2}-k\left|\left\|w\left|\left\|_{1}^{2} \leqslant \nu\left|\left\|w\left|\left\|_{2}^{2}+C_{2} \max _{i=\mathrm{I}, \mathrm{II}}\left(\left|\left\|v^{i}+z\left|\left\|_{2}\left|\left\|v^{i}+z \mid\right\|_{1}\right)^{2}\left|\|w \mid\|_{1}^{2}\right.\right.\right.\right.\right.\right.\right.\right.\right.\right.\right.\right.\right.\right.\right.\right.\right.\right.$.

Тогда

$$
\frac{1}{2} \frac{\partial}{\partial t}\left\langle A_{1} w, w\right\rangle \leqslant C_{3}\left(1+\max _{i=\mathrm{I}, \mathrm{II}}\left(\left|\left\|v^{i}+z\left|\left\|_{2}\right\| v^{i}+z\right|\right\|_{1}\right)^{2}\right)\left|\|w \mid\|_{1}^{2},\right.\right.
$$

где $C_{3}=\max \left\{C_{2}, k\right\}$. В силу $(2.1)$, определения операторов $A_{0}$ и $A_{1}$, так как $\gamma \geqslant 0$, справедливо неравенство

$$
0 \leqslant \mid\|\psi\|_{1}^{2}=\left\langle A_{0} \psi, \psi\right\rangle \leqslant\left\langle A_{1} \psi, \psi\right\rangle \quad \text { для любого } \psi \in H^{1} .
$$

Поэтому

$$
\frac{1}{2} \frac{\partial}{\partial t}\left\langle A_{1} w, w\right\rangle \leqslant C_{3}\left(1+\max _{i=\mathrm{I}, \mathrm{II}}\left(\left|\left\|v^{i}+z\left|\left\|_{2}\left|\left\|v^{i}+z \mid\right\|_{1}\right)^{2}\right)\left\langle A_{1} w, w\right\rangle .\right.\right.\right.\right.\right.
$$

5 Математический сборник, т. 203, вып. 10 
Интегрируя по $t$ последнее неравенство и учитывая, что $w(0)=0$, получим

$$
\left\langle A_{1} w(t), w(t)\right\rangle \leqslant \int_{0}^{t} \widetilde{\psi}(s)\left\langle A_{1} w(s), w(s)\right\rangle d s,
$$

где

$$
\widetilde{\psi}(s)=2 C_{3}\left(1+\max _{i=\mathrm{I}, \mathrm{II}}\left(\left|\left\|v^{i}+z\left|\left\|_{2}\left|\left\|v^{i}+z \mid\right\|_{1}\right)^{2}\right)>0 .\right.\right.\right.\right.\right.
$$

Отсюда, домножив на $\widetilde{\psi}(t) \exp \left(-\int_{0}^{t} \widetilde{\psi}(s) d s\right)$ левую и правую часть неравен-
ства, имеем

$$
\begin{aligned}
\frac{d}{d t}(\exp ( & \left.\left.-\int_{0}^{t} \widetilde{\psi}(s) d s\right) \int_{0}^{t} \widetilde{\psi}(s)\left\langle A_{1} w(s), w(s)\right\rangle d s\right)=\widetilde{\psi}(t) \exp \left(-\int_{0}^{t} \widetilde{\psi}(s) d s\right) \\
& \times\left(\left\langle A_{1} w(t), w(t)\right\rangle-\int_{0}^{t} \widetilde{\psi}(s)\left\langle A_{1} w(s), w(s)\right\rangle d s\right) \leqslant 0 .
\end{aligned}
$$

Интегрируя по $t$, получим

$$
\exp \left(-\int_{0}^{t} \widetilde{\psi}(s) d s\right) \int_{0}^{t} \widetilde{\psi}(s)\left\langle A_{1} w(s), w(s)\right\rangle d s \leqslant 0 \quad \text { для любого } t \in[0, T] .
$$

Поэтому $\left\langle A_{1} w(t), w(t)\right\rangle=0$ для почти всех $t \in[0, T]$. Поскольку очевидно, что оператор $A_{1}$ положительно определен, то $w(t)=0$ для почти всех $t \in[0, T]$.

Единственность доказана.

Существование. Для доказательства существования будем использовать общий подход, называемый методом Галеркина, т.е. построим приближенные решения задачи Коши (3.7) на $[0, T] \times S$ и покажем, что они имеют предел, который удовлетворяет исходной задаче Коши $(3.7)$ на $[0, T] \times S$. Доказательство проведем в три этапа. Заметим, что предлагаемая схема является аналогом схемы доказательства теоремы 2.1.13 в [24] существования решения для уравнения Навье-Стокса по переменным $x$ в двумерной ограниченной области или двумерном торе с условием того, что решение и правая часть системы представляют собой бездивергентное векторное поле по переменным $x$.

Шаг 1: априорная оценка. Пусть $v$ - решение задачи Коши $(3.7)$ на $[0, T] \times S$. Вычислим вдоль этой траектории уравнения в (3.7) производную функции $\left\|A_{1} v\right\|_{0}^{2}$. В силу (2.1) и леммы 1.2 из [25; гл. III, § 1.4]

$$
\begin{gathered}
\frac{1}{2} \frac{\partial}{\partial t}\|\| A_{1} v \mid \|_{0}^{2}=\left\langle\frac{\partial}{\partial t} A_{1} v, A_{1} v\right\rangle=\left\langle-\nu A_{2} v-A_{3} v-B(v+z)+f, A_{1} v\right\rangle \\
=-\nu\left\langle A_{2} v, A_{1} v\right\rangle-\left\langle A_{3} v, A_{1} v\right\rangle-\left\langle B(v+z), A_{1} v\right\rangle+\left\langle f, A_{1} v\right\rangle .
\end{gathered}
$$

Оценим сверху $\left|\left\langle B(v+z), A_{1} v\right\rangle\right|$. По определению $B(u)$ и лемме 3

$$
\begin{aligned}
&\left|\left\langle B(v+z), A_{1} v\right\rangle\right|\left|\left\langle\left(\begin{array}{c}
J\left(\Delta\left(v_{1}+z_{1}\right)+2 \mu, v_{1}+z_{1}\right)+J\left(\Delta\left(v_{2}+z_{2}\right), v_{2}+z_{2}\right) \\
J\left(\Delta\left(v_{2}+z_{2}\right)-\gamma\left(v_{2}+z_{2}\right), v_{1}+z_{1}\right)+J\left(\Delta\left(v_{1}+z_{1}\right)+2 \mu, v_{2}+z_{2}\right)
\end{array}\right), A_{1} v\right\rangle\right| \\
&=\mid-\left(J\left(\Delta z_{1}, v_{1}+z_{1}\right), \Delta v_{1}\right)-2\left(J\left(\mu, z_{1}\right), \Delta v_{1}\right)-\left(J\left(\Delta z_{2}, v_{2}+z_{2}\right), \Delta v_{1}\right) \\
& \quad-\left(J\left(\Delta z_{2}-\gamma\left(v_{2}+z_{2}\right), v_{1}+z_{1}\right), \Delta v_{2}\right)-\left(J\left(\Delta z_{1}, v_{2}+z_{2}\right), \Delta v_{2}\right) \\
& \quad-2\left(J\left(\mu, z_{2}\right), \Delta v_{2}\right)+\gamma\left(J\left(\Delta\left(v_{2}+z_{2}\right)-\gamma z_{2}, v_{1}+z_{1}\right), v_{2}\right) \\
& \quad+\gamma\left(J\left(\Delta\left(v_{1}+z_{1}\right), z_{2}\right), v_{2}\right)+2 \gamma\left(J\left(\mu, z_{2}\right), v_{2}\right) \mid .
\end{aligned}
$$


Отсюда по лемме 1, пп. II и III, равенству (3.13) и ввиду непрерывности вложения $h^{2}$ в $h^{0}$ получим

$$
\begin{aligned}
\left|\left\langle B(v+z), A_{1} v\right\rangle\right| \leqslant & C_{4}\left(( | | | z | \| _ { 3 } | | | z | \| _ { 2 } | | | v | \| _ { 2 } | | | v | \| _ { 1 } ) ^ { 1 / 2 } \left|\|v \mid\|_{3}\right.\right. \\
& +\left|\left\|z \left|\left\|_ { 2 } \left(\left|\left\|z \left|\left\|_ { 3 } | \| z | \| _ { 1 } ) ^ { 1 / 2 } \left|\left\|v \left|\left\|_{3}+\left|\left\|z \left|\left\|_{1}\left|\|v \mid\|_{2}\right) .\right.\right.\right.\right.\right.\right.\right.\right.\right.\right.\right.\right.\right.\right.\right.\right.\right.
\end{aligned}
$$

Везде ниже в шаге 1 считаем, что $C_{i} \geqslant 0, i \geqslant 4,-$ константы, не зависящие от $\psi \in H^{1}$ (если в оценке участвует это векторное поле), $v, z$. Тогда из непрерывности вложения $h^{2}$ в $h^{1}$ и очевидного неравенства

$$
a b \leqslant \frac{1}{2}\left(a^{2}+b^{2}\right), \quad a, b \in \mathbb{R},
$$

вытекает

$\left|\left\langle B(v+z), A_{1} v\right\rangle\right| \leqslant \frac{\nu}{4}\left|\left\|v\left|\left\|_{3}^{2}+\left(1+C_{5}\left|\left\|z\left|\left\|\left.\right|_{3}\left|\|z \mid\|_{2}\right)\left|\|v \mid\|_{2}^{2}+C_{5}\left(\left|\left\|z\left|\left\|_{3}\left|\left\|z\left|\left\|_{2}^{3}+\left|\|z \mid\|_{1}^{2}\right)\right.\right.\right.\right.\right.\right.\right.\right.\right.\right.\right.\right.\right.\right.\right.\right.\right.\right.\right.$.

Из (2.1) и (3.18) имеем

$$
\left\langle A_{2} v, A_{1} v\right\rangle=\left\langle A_{1} A_{0} v, A_{0} v\right\rangle \geqslant\left|\left\|A_{0} v \mid\right\|_{1}^{2}=\|\| v \|_{3}^{2} .\right.
$$

Ввиду (2.1), непрерывности вложения $h^{2}$ в $h^{0}, h^{1}$, оценки (3.16) и неравенств $\nu>0, \gamma, \rho, k, k_{1} \geqslant 0$

$$
\begin{aligned}
\left\langle A_{3} v, A_{1} v\right\rangle & =\left\langle A_{3} A_{0}^{1 / 2} v, A_{0}^{1 / 2} v\right\rangle+\gamma\left(k\left\langle\Delta v_{1}, v_{2}\right\rangle+\left(2 k+k_{1}+\nu \gamma\right)\left\|v_{2}\right\|_{1}^{2}+\rho\left\|v_{2}\right\|_{0}^{2}\right) \\
& \geqslant-C_{6} \mid\|v\| \|_{2}^{2} .
\end{aligned}
$$

Тогда из $(2.2),(3.20)$ и $(3.22)$ получим

$$
\begin{aligned}
\frac{1}{2} \frac{\partial}{\partial t}\left|\left\|A_{1} v \mid\right\|_{0}^{2} \leqslant-\right. & \frac{3 \nu}{4}\left|\left\|v \left|\left\|_{3}^{2}+\left(1+C_{6}+C_{5}\left|\left\|z\left|\left\|_{3}\left|\|z \mid\|_{2}\right)\right\|\right|\right\| v\right| \|_{2}^{2}\right.\right.\right.\right.\right. \\
& +C_{5}\left(\left|\left\|z \left|\left\|_ { 3 } \left|\left\|z \left|\left\|_{2}^{3}+\left|\|z \mid\|_{1}^{2}\right)+\left|\left\|f \left|\left\|_ { - 1 } \left|\left\|A_{1} v \mid\right\|_{1} .\right.\right.\right.\right.\right.\right.\right.\right.\right.\right.\right.\right.\right.\right.
\end{aligned}
$$

Поскольку $\gamma \geqslant 0$, то

$$
\left\|v \left|\left\|_{2}^{2} \leqslant\left|\left\|A_{1} v \mid\right\|_{0}^{2} .\right.\right.\right.\right.
$$

В силу (2.1) и непрерывности вложения $h^{3}$ в $h^{1}, h^{2}$

$$
\left\|v \left|\left\|_{3}^{2} \leqslant\left|\left\|A _ { 1 } v \left|\left\|_{1}^{2}=\left\langle A_{1}^{2} v, A_{0} v\right\rangle=\left|\left\|v \left|\| _ { 3 } ^ { 2 } + 2 \gamma \| v _ { 2 } \| _ { 2 } ^ { 2 } + \gamma ^ { 2 } \| v _ { 2 } \left\|_{1}^{2} \leqslant C_{7}\left|\|v \mid\|_{3}^{2} .\right.\right.\right.\right.\right.\right.\right.\right.\right.\right.\right.\right.
$$

Поэтому ввиду (3.21)

$$
\begin{aligned}
& \frac{\partial}{\partial t}\left|\left\|A _ { 1 } v \left|\left\|_{0}^{2} \leqslant-\nu\left|\left\|v \left|\left\|_{3}^{2}+2\left(1+C_{6}+C_{5}\left|\left\|z \left|\left\|_ { 3 } | \| z | \| _ { 2 } ) \left|\left\|A_{1} v \mid\right\|_{0}^{2}\right.\right.\right.\right.\right.\right.\right.\right.\right.\right.\right.\right.\right.\right. \\
& +2 C_{5}\left(| | | z | \left\|_ { 3 } \left|\left\|z\left|\left\|_{2}^{3}+|||z|\right\|_{1}^{2}\right)+\frac{2 C_{7}}{\nu}\left|\|f \mid\|_{-1}^{2} .\right.\right.\right.\right.\right.
\end{aligned}
$$

Тогда

где

$$
\frac{\partial}{\partial t}\left|\left\|A _ { 1 } v \left|\left\|_{0}^{2}-\widetilde{\psi}(t)\left|\left\|A _ { 1 } v \left|\left\|_{0}^{2}+\nu\left|\|v \mid\|_{3}^{2} \leqslant \widetilde{\theta}(t)\right.\right.\right.\right.\right.\right.\right.\right.\right.
$$

$$
\begin{gathered}
\widetilde{\psi}(t)=2\left(1+C_{6}+C_{5}\left|\left\|z \left|\left\|_{3}\left|\|z \mid\|_{2}\right)>0\right.\right.\right.\right.\right. \\
\widetilde{\theta}(t)=2 C_{5}\left(\left|\left\|z \left|\left\|_ { 3 } \left|\left\|z \left|\left\|_{2}^{3}+\left|\|z \mid\|_{1}^{2}\right)+\frac{2 C_{7}}{\nu}\left|\|f \mid\|_{-1}^{2} .\right.\right.\right.\right.\right.\right.\right.\right.\right.\right.
\end{gathered}
$$


Интегрируя неравенство (3.27) по $t$, получим $\left|\left\|A_{1} v(t)\left|\left\|_{0}^{2}-\left|\left\|A_{1} v(0)\left|\left\|_{0}^{2}-\int_{0}^{t} \widetilde{\psi}(s)\left|\left\|A_{1} v(s)\left|\left\|_{0}^{2} d s+\nu \int_{0}^{t}\left|\|v(s) \mid\|_{3}^{2} d s \leqslant \int_{0}^{t} \tilde{\theta}(s) d s\right.\right.\right.\right.\right.\right.\right.\right.\right.\right.\right.\right.\right.$. Отсюда, домножив на $\widetilde{\psi}(t) \exp \left(-\int_{0}^{t} \widetilde{\psi}(s) d s\right)$ левую и правую часть неравенства, используя (3.19) с заменой $\left\langle A_{1} w, w\right\rangle$ на $\left.\left|\| A_{1} v\right|\right|_{0} ^{2}$, имеем

$$
\begin{aligned}
& \frac{d}{d t}\left(\exp \left(-\int_{0}^{t} \widetilde{\psi}(s) d s\right) \int_{0}^{t} \widetilde{\psi}(s) \mid\left\|A_{1} v(s)\right\|_{0}^{2} d s\right) \\
& \quad \leqslant \widetilde{\psi}(t) \exp \left(-\int_{0}^{t} \widetilde{\psi}(s) d s\right)\left(\left\|A_{1} v(0)\right\|_{0}^{2}+\int_{0}^{t} \widetilde{\theta}(s) d s-\nu \int_{0}^{t} \mid\|v(s)\|_{3}^{2} d s\right) .
\end{aligned}
$$

Проинтегрируем по $t$ и домножим неравенство на $\exp \left(\int_{0}^{t} \widetilde{\psi}(s) d s\right)$ :

$$
\int_{0}^{t} \widetilde{\psi}(s)\left|\left\|A_{1} v(s) \mid\right\|_{0}^{2} d s \leqslant \int_{0}^{t} \widetilde{\psi}(\xi) \exp \left(\int_{\xi}^{t} \widetilde{\psi}(s) d s\right)\left(\left|\left\|A_{1} v(0) \mid\right\|_{0}^{2}+\int_{0}^{\xi} \widetilde{\theta}(s) d s\right) d \xi .\right.\right.
$$

Тогда из (3.28) выводим для любого $t \in[0, T]$

$$
\begin{aligned}
\left\|A_{1} v(t) \mid\right\|_{0}^{2} & +\nu \int_{0}^{t}\left|\left\|v ( s ) \left|\left\|_{3}^{2} d s \leqslant \mid\right\| A_{1} v(0) \|_{0}^{2}+\int_{0}^{t} \widetilde{\theta}(s) d s\right.\right.\right. \\
& +\int_{0}^{t} \widetilde{\psi}(\xi) \exp \left(\int_{\xi}^{t} \widetilde{\psi}(s) d s\right)\left(\left|\left\|A_{1} v(0) \mid\right\|_{0}^{2}+\int_{0}^{\xi} \widetilde{\theta}(s) d s\right) d \xi .\right.
\end{aligned}
$$

Нетрудно показать, что

$$
\int_{0}^{t} \widetilde{\psi}(\xi) \exp \left(\int_{\xi}^{t} \widetilde{\psi}(s) d s\right) d \xi=\exp \left(\int_{0}^{t} \widetilde{\psi}(s) d s\right)-1
$$

Поэтому в силу $(3.25)$ и того, что $v(0)=u_{0}$ и $\widetilde{\psi}(t)>0, \widetilde{\theta}(t) \geqslant 0, t \in[0, T]$,

$$
\left\|v ( t ) \left|\left\|_{2}^{2}+\nu \int_{0}^{t}\left|\|v(s) \mid\|_{3}^{2} d s \leqslant\left(\left\|\mid A_{1} u_{0}\right\|_{0}^{2}+\int_{0}^{T} \tilde{\theta}(s) d s\right) \exp \left(\int_{0}^{T} \widetilde{\psi}(s) d s\right) .\right.\right.\right.\right.
$$

Следовательно, ввиду условий теоремы на $u_{0}, z, f$ и непрерывности вложения $\Xi_{T}$ в $C\left(0, T ; H^{2}\right)$

$$
\|v\|_{C\left(0, T ; H^{2}\right)},\|v\|_{L_{2}\left(0, T ; H^{3}\right)} \leqslant \widetilde{C}_{1}\left(u_{0}, z, f\right)<\infty
$$

где $\widetilde{C}_{1}\left(u_{0}, z, f\right) \geqslant 0$ - константа, зависящая от $u_{0}, z, f$ и не зависящая от $v$.

Для получения оценки сверху на $\left\|\frac{\partial}{\partial t} v\right\|_{L_{2}\left(0, T ; H^{1}\right)}$ вначале получим оценки сверху на $\|B(v+z)\|_{L_{2}\left(0, T ; H^{-1}\right)}$ и $\left\|\nu A_{2} v+A_{3} v\right\|_{L_{2}\left(0, T ; H^{-1}\right)}$.

Поскольку для любого $\psi \in H^{1}$ в силу леммы 1 , п. II, непрерывности вложений $h^{3}$ в $h^{1}$ и $h^{2}$ в $h^{0}$, определения $B(u)$, так как $\mu \in C^{\infty}(S)$,

$|\langle B(v+z), \psi\rangle| \leqslant C_{8}\left(|||v+z|\left\|_{2}|||v+z|\right\|_{1}\left(C_{8}+|||v+z| \|_{3}\right)\left(C_{8}+\left|\|v+z \mid\|_{2}\right)\right)^{1 / 2}\left|\|\psi \mid\|_{1}\right.\right.$, 
то по лемме 1.5 из [6; гл. I, §3] и непрерывности вложений $h^{3}$ в $h^{2}$ и $h^{2}$ в $h^{1}$

$$
\|B(v+z)\|_{L_{2}\left(0, T ; H^{-1}\right)}^{2} \leqslant C_{9} \int_{0}^{T} \mid\|v(t)+z(t)\| \|_{2}^{2}\left(C_{9}+\left|\|v(t)+z(t) \mid\|_{3}^{2}\right) d t .\right.
$$

Отсюда ввиду (3.30), непрерывности вложения $h^{2}$ в $h^{1}$ и того, что $z \in \varkappa_{T}$,

$$
\|B(v+z)\|_{L_{2}\left(0, T ; H^{-1}\right)} \leqslant \widetilde{C}_{2}\left(u_{0}, z, f\right)<\infty,
$$

где $\widetilde{C}_{2}\left(u_{0}, z, f\right) \geqslant 0$ - константа, зависящая от $u_{0}, z, f$ и не зависящая от $v$.

Из (2.1) и непрерывности вложений $h^{1}$ в $h^{0}, h^{3}$ в $h^{0}, h^{1}, h^{2}$ для любого $\psi \in H^{1}$ имеем

$$
\begin{aligned}
\left|\left\langle\nu A_{2} v+A_{3} v, \psi\right\rangle\right| & =\left|\nu\left\langle A_{0}^{3 / 2} v, A_{0}^{1 / 2} \psi\right\rangle+\left\langle A_{3} v, \psi\right\rangle\right| \\
& \leqslant \nu\left|\left\|v\left|\left\|_{3}\left|\|\psi\|_{1}+\right|\right\| A_{3} v\right|\right\|_{0}\right|\left\|\psi \left|\left\|_{0} \leqslant C_{10}\left|\left\|v \left|\left\|_{3} \mid\right\| \psi \|_{1} .\right.\right.\right.\right.\right.\right.
\end{aligned}
$$

Поэтому в силу оценки (3.30) и по лемме 1.5 из [6; гл. I, §3]

$$
\left\|\nu A_{2} v+A_{3} v\right\|_{L_{2}\left(0, T ; H^{-1}\right)} \leqslant C_{10}\|v\|_{L_{2}\left(0, T ; H^{3}\right)} \leqslant C_{10} \widetilde{C}_{1}\left(u_{0}, z, f\right)<\infty .
$$

Поскольку $v$ - решение уравнения из (3.7), то из (3.31) и (3.32) получим

$$
\left\|\frac{\partial}{\partial t} A_{1} v\right\|_{L_{2}\left(0, T ; H^{-1}\right)} \leqslant C_{10} \widetilde{C}_{1}\left(u_{0}, z, f\right)+\widetilde{C}_{2}\left(u_{0}, z, f\right)+\|f\|_{L_{2}\left(0, T ; H^{-1}\right)}=\widetilde{C}_{3}\left(u_{0}, z, f\right) .
$$

Тогда ввиду перестановочности производных в смысле распределений (см., например, [26; §1.2, замечание 1 , и §3.6] и [6; гл. I, §3])

$$
\left\|\frac{\partial}{\partial t} v\right\|_{L_{2}\left(0, T ; H^{1}\right)} \leqslant \widetilde{C}_{3}\left(u_{0}, z, f\right) .
$$

Следовательно,

$$
\|v\|_{\Xi_{T}} \leqslant \widetilde{C}_{4}\left(u_{0}, z, f\right),
$$

$\widetilde{C}_{4}\left(u_{0}, z, f\right)=\max \left\{\widetilde{C}_{1}\left(u_{0}, z, f\right), \widetilde{C}_{3}\left(u_{0}, z, f\right)\right\}$.

Шаг 2: приближенные решения. Обозначим через $H_{N}$ линейную оболочку множества $\left\{E_{m}^{n}\right\}_{1 \leqslant m \leqslant 4 n+2}^{1 \leqslant n \leqslant N}$ собственных векторных полей оператора $A_{0}$ и через $P_{N}: H^{-1} \rightarrow H^{N}$ - ортогональный проектор на множество $H_{N}$ (определение и свойства ортогонального проектора см., например, в $[23 ; \S 6]$ и [27; гл. VII, § 2]).

Определим приближенное решение следующим образом. Подействовав формально оператором $P_{N}$ на уравнение в $(3.7)$, получим

$$
P_{N} \frac{\partial}{\partial t} A_{1} v+\nu P_{N} A_{2} v+P_{N} A_{3} v+P_{N} B(v+z)=P_{N} f, \quad t>0 .
$$

Очевидно, что векторное поле $v \in H_{N}$ удовлетворяет этому уравнению тогда и только тогда, когда

$$
\frac{\partial}{\partial t} A_{1} v+\nu A_{2} v+A_{3} v+P_{N} B(v+z)=P_{N} f, \quad t>0 .
$$

Уравнение дополним начальным условием

$$
v(0)=P_{N} u_{0}
$$


Будем считать $v$ решением задачи Коши (3.34), (3.35), если $v \in C\left(0, T ; H_{N}\right)$, $\frac{\partial}{\partial t} v \in L_{2}\left(0, T ; H_{N}\right)$, выполнено $(3.35)$ и уравнение (3.34) имеет место в пространстве $L_{2}\left(0, T ; H_{N}\right)$.

Пусть существует единственное решение $v=v_{N}$ задачи Коши (3.34), (3.35). Вычислим производную функции \|\|$A_{1} v_{N} \|_{0}^{2}$ вдоль траектории (3.34), (3.35). В силу леммы 1.2 из [25; гл. III, § 1.4] и $(2.1)$

$$
\begin{aligned}
\frac{1}{2} \frac{\partial}{\partial t} & \left\|A_{1} v_{N} \mid\right\|_{0}^{2}=\left\langle\frac{\partial}{\partial t} A_{1} v_{N}, A_{1} v_{N}\right\rangle \\
& =\left\langle-\nu A_{2} v_{N}-A_{3} v_{N}-P_{N} B\left(v_{N}+z\right)+P_{N} f, A_{1} v_{N}\right\rangle \\
& =\left\langle-\nu A_{2} v_{N}-A_{3} v_{N}-B\left(v_{N}+z\right)+f, A_{1} v_{N}\right\rangle
\end{aligned}
$$

Тогда, повторяя рассуждения шага 1, учитывая небольшие отличия в силу того, что $v_{N}$ удовлетворяет задаче Коши (3.34), (3.35) с $P_{N}$, используя свойства ортогонального проектора, получим, что выполнена оценка (3.30)

$$
\left\|v_{N}\right\|_{C\left(0, T ; H^{2}\right)} \leqslant \widetilde{C}_{1}\left(u_{0}, z, f\right)
$$

и оценка (3.33)

$$
\left\|v_{N}\right\|_{\Xi_{T}} \leqslant \widetilde{C}_{4}\left(u_{0}, z, f\right) .
$$

Из задачи Коши (3.34), (3.35) следует, что ее решение сводится к решению задачи Коши для нелинейной системы обыкновенных дифференциальных уравнений первого порядка

$$
\frac{d}{d t} y=\vartheta(t, y), \quad y=\left(y_{1}, y_{2}, \ldots, y_{2 N(N+2)}\right)^{\mathrm{T}},
$$

где $2 N(N+2)$ - размерность $H_{N}$, векторное поле $\vartheta(t, \cdot)=\left(\vartheta_{1}(t, \cdot), \vartheta_{2}(t, \cdot), \ldots\right.$, $\left.\vartheta_{2 N(N+2)}(t, \cdot)\right)^{\mathrm{T}}$ удовлетворяет условиям Каратеодори из [28; гл. I, § 1.1] и условию (6) теоремы 2 из [28; гл. I, §1.2] в силу (3.36) и так как $z \in \varkappa_{T}, f \in$ $L_{2}\left(0, T ; H^{-1}\right)$. Ввиду (3.36) имеет место априорная оценка $\|y\|_{C\left(0, T ; \mathbb{R}^{2 N(N+2)}\right)} \leqslant$ $C_{11}$, где $C_{11} \geqslant 0$ - константа. Тогда в силу теорем 1,2 из [28; гл. I, § 1.2$]$ и теоремы 4 из [28; гл. I, §1.3] (общей теории уравнений Каратеодори - обыкновенных дифференциальных уравнений с разрывной правой частью) следует, что эта задача Коши однозначно разрешима на интервале $[0, T]$, причем $y \in C\left(0, T ; \mathbb{R}^{2 N(N+2)}\right)$ и $\frac{\partial}{\partial t} y \in L_{2}\left(0, T ; \mathbb{R}^{2 N(N+2)}\right)$. Поэтому задача Коши (3.34), (3.35) однозначно разрешима, $v=v_{N}$ на интервале $[0, T]$, уравнение (3.34) имеет место в пространстве $L_{2}\left(0, T ; H_{N}\right)$ и $v_{N} \in C\left(0, T ; H_{N}\right), \frac{\partial}{\partial t} v_{N} \in L_{2}\left(0, T ; H_{N}\right)$.

Из (3.37) следует, что последовательность $\left\{v_{N}\right\}_{N=1}^{\infty}$ ограничена в $\Xi_{T}$.

Шаг 3: предельный переход. Поскольку замкнутый шар в гильбертовом пространстве слабо компактен (см., например, учитывая рефлексивность гильбертова пространства, [22; гл. IV,$\S 3.3$, замечание, и § 3.4 , теорема 5]), линейные операторы

$$
\begin{array}{rlrl}
\Xi_{T} & \rightarrow L_{2}\left(0, T ; H^{-1}\right), \quad \psi \mapsto \frac{\partial}{\partial t} A_{1} \psi ; & \Xi_{T} \rightarrow L_{2}\left(0, T ; H^{1}\right), & \psi \mapsto \frac{\partial}{\partial t} \psi ; \\
\Xi_{T} & \rightarrow L_{2}\left(0, T ; H^{-1}\right), \quad \psi \mapsto A_{0}^{2} \psi ; & \Xi_{T} \rightarrow L_{2}\left(0, T ; H^{3}\right), \quad \psi \mapsto \psi ;
\end{array}
$$

ограничены, $\Xi_{T}$ компактно вложено в $L_{2}\left(0, T ; H^{2}\right)$ (см., например, [15; гл. I, $\S 5.2$, теорема 5.1]), оператор обобщенного дифференцирования слабо замкнут 
(см., например, [29; §5, теорема 1]), то ввиду [22; гл. II, §2.1, §2.2, теорема 2, $\S 6.1$, теорема 2] и того, что из последовательности, сходящейся в среднем, можно извлечь подпоследовательность, сходящуюся почти всюду (см., например, $\left[22\right.$; гл. VII, §2.5, свойство 4]), вложения $h^{2}$ в $h^{1}, h^{0}$ непрерывны и имеет место (2.3), из ограниченной последовательности $\left\{v_{N}\right\}_{N=1}^{\infty}$ в $\Xi_{T}$ можно выделить подпоследовательность такую, что при $i \rightarrow \infty$

$$
\begin{gathered}
v_{N_{i}} \rightarrow v \quad \text { слабо в } \Xi_{T} \\
\frac{\partial}{\partial t} A_{1} v_{N_{i}} \rightarrow \frac{\partial}{\partial t} A_{1} v, \quad A_{0}^{2} v_{N_{i}} \rightarrow A_{0}^{2} v \quad \text { слабо в } L_{2}\left(0, T ; H^{-1}\right) \\
A_{3} v_{N_{i}} \rightarrow A_{3} v \quad \text { слабо в } L_{2}\left(0, T ; H^{1}\right), \\
v_{N_{i}} \rightarrow v \quad \text { сильно в } L_{2}\left(0, T ; H^{2}\right), \\
v_{N_{i}} \rightarrow \quad \nabla v_{N_{i}} \rightarrow \nabla v, \Delta v_{N_{i}} \rightarrow \Delta v \quad \text { почти всюду } \\
\frac{\partial}{\partial t} v_{N_{i}} \rightarrow \frac{\partial}{\partial t} v \quad \text { слабо в } L_{2}\left(0, T ; H^{1}\right), \quad v_{N_{i}} \rightarrow v \quad \text { слабо в } L_{2}\left(0, T ; H^{3}\right) .
\end{gathered}
$$

Заметим, что из непрерывности вложений $\Xi_{T}$ в $C\left(0, T ; H^{2}\right), h^{3}$ в $h^{2}, h^{2}$ в $h^{1}, h^{0}$, леммы 4, равенства (3.13), сходимости почти всюду в (3.40), леммы 1.3 из [15; гл. I, § 1.4] и того, что $z \in \varkappa_{T}, v_{N_{i}}, v \in \Xi_{T}$, следует

$$
B\left(v_{N_{i}}+z\right) \rightarrow B(v+z) \quad \text { слабо в } L_{2}\left(0, T ; H^{-1}\right) .
$$

Пусть $j \geqslant 1$ такое, что $N_{i} \geqslant j$. Подействуем оператором $P_{j}$ на уравнение (3.34) при $N=N_{i}$. Ввиду $P_{j} P_{N_{i}}=P_{j}$ имеем

$$
P_{j} \frac{\partial}{\partial t} A_{1} v_{N_{i}}+\nu P_{j} A_{2} v_{N_{i}}+P_{j} A_{3} v_{N_{i}}+P_{j} B\left(v_{N_{i}}+z\right)=P_{j} f, \quad t>0 .
$$

Тогда в силу (3.38)-(3.40), (3.42) получим

$$
P_{j} \frac{\partial}{\partial t} A_{1} v+\nu P_{j} A_{2} v+P_{j} A_{3} v+P_{j} B(v+z)=P_{j} f, \quad t>0,
$$

где равенство имеет место в пространстве $L_{2}\left(0, T ; H^{-1}\right)$. Так как $j$ - произвольно, то в $L_{2}\left(0, T ; H^{-1}\right)$

$$
\frac{\partial}{\partial t} A_{1} v+\nu A_{2} v+A_{3} v+B(v+z)=f, \quad t>0 .
$$

Пусть $\psi$ - произвольная бесконечно дифференцируемая функция из $[0, T]$ в $H^{-1}$ такая, что $\psi(T)=0$. По формуле интегрирования по частям (ее доказательство легко получить, используя свойство (1.71) из [25; гл. III, § 1.4] при $\left.u=A_{1} v\right)$ в силу непрерывности вложения $\Xi_{T}$ в $C\left(0, T ; H^{2}\right)$ имеем

$$
\int_{0}^{T}\left\langle\frac{\partial}{\partial t} v, \psi\right\rangle d t=-\langle v(0), \psi(0)\rangle-\int_{0}^{T}\left\langle v, \frac{\partial}{\partial t} \psi\right\rangle d t
$$

и, учитывая (3.35),

$$
\int_{0}^{T}\left\langle\frac{\partial}{\partial t} v_{N_{i}}, \psi\right\rangle d t=-\left\langle P_{N_{i}} u_{0}, \psi(0)\right\rangle-\int_{0}^{T}\left\langle v_{N_{i}}, \frac{\partial}{\partial t} \psi\right\rangle d t
$$


Поскольку $P_{N_{i}} u_{0} \rightarrow u_{0}$ сильно в $H^{2}$, то $P_{N_{i}} u_{0} \rightarrow u_{0}$ слабо в $H^{2}$. Поэтому, переходя в последнем равенстве к пределу, получим в силу (3.41)

$$
\int_{0}^{T}\left\langle\frac{\partial}{\partial t} v, \psi\right\rangle d t=-\left\langle u_{0}, \psi(0)\right\rangle-\int_{0}^{T}\left\langle v, \frac{\partial}{\partial t} \psi\right\rangle d t .
$$

Тогда из (3.43) и (3.44) следует, что

$$
\left\langle u_{0}, \psi(0)\right\rangle=\langle v(0), \psi(0)\rangle
$$

Ввиду произвольности $\psi(0)$ выводим $v(0, x)=u_{0}(x)$ для почти всех $x \in S$.

Существование решения доказано.

$\Phi$ равномерно непрерывен по Липшицу на ограниченных подмножествах. В определении 3 в нашем случае $j=7$. Пусть $v^{\mathrm{I}}=\Phi\left(\nu^{\mathrm{I}}, \gamma^{\mathrm{I}}, \rho^{\mathrm{I}}, k^{\mathrm{I}}, k_{1}^{\mathrm{I}}, f^{\mathrm{I}}, u_{0}^{\mathrm{I}}, z^{\mathrm{I}}\right)$, $v^{\mathrm{II}}=\Phi\left(\nu^{\mathrm{II}}, \gamma^{\mathrm{II}}, \rho^{\mathrm{II}}, k^{\mathrm{II}}, k_{1}^{\mathrm{II}}, f^{\mathrm{II}}, u_{0}^{\mathrm{II}}, z^{\mathrm{II}}\right), w=v^{\mathrm{I}}-v^{\mathrm{II}}$. Тогда $w$ удовлетворяет следующей задаче Коши:

$$
\begin{aligned}
\frac{\partial}{\partial t} A_{1}^{\mathrm{I}} w+ & \nu^{\mathrm{I}} A_{2} w+A_{3}^{\mathrm{I}} w+B^{\mathrm{I}}\left(v^{\mathrm{I}}+z^{\mathrm{I}}\right)-B^{\mathrm{I}}\left(v^{\mathrm{II}}+z^{\mathrm{II}}\right) \\
= & f^{\mathrm{I}}-f^{\mathrm{II}}-\frac{\partial}{\partial t}\left(A_{1}^{\mathrm{I}}-A_{1}^{\mathrm{II}}\right) v^{\mathrm{II}}-\left(\nu^{\mathrm{I}}-\nu^{\mathrm{II}}\right) A_{2} v^{\mathrm{II}}-\left(A_{3}^{\mathrm{I}}-A_{3}^{\mathrm{II}}\right) v^{\mathrm{II}} \\
& \quad-\left(B^{\mathrm{I}}\left(v^{\mathrm{II}}+z^{\mathrm{II}}\right)-B^{\mathrm{II}}\left(v^{\mathrm{II}}+z^{\mathrm{II}}\right)\right), \quad t>0, \quad w(0)=u_{0}^{\mathrm{I}}-u_{0}^{\mathrm{II}},
\end{aligned}
$$

где $A_{1}^{\mathrm{I}}, A_{1}^{\mathrm{II}}$ - операторы $A_{1}$ с параметрами соответственно $\gamma^{\mathrm{I}}, \gamma^{\mathrm{II}} ; A_{3}^{\mathrm{I}}, A_{3}^{\mathrm{II}}$ - операторы $A_{3}$ с параметрами соответственно $\left(\nu^{\mathrm{I}}, \gamma^{\mathrm{I}}, \rho^{\mathrm{I}}, k^{\mathrm{I}}, k_{1}^{\mathrm{I}}\right)$ и $\left(\nu^{\mathrm{II}}, \gamma^{\mathrm{II}}, \rho^{\mathrm{II}}, k^{\mathrm{II}}, k_{1}^{\mathrm{II}}\right)$; $B^{\mathrm{I}}(u), B^{\mathrm{II}}(u)$ - операторы $B(u)$ с параметрами соответственно $\gamma^{\mathrm{I}}, \gamma^{\mathrm{II}}$. В силу оценок (3.29) и (3.33) $\max _{q=\mathrm{I}, \mathrm{II}}\left\|v^{q}\right\|_{C\left(0, T ; H^{2}\right)}, \max _{q=\mathrm{I}, \mathrm{II}}\left\|v^{q}\right\|_{\Xi_{T}}$ будут ограничены константой, зависящей от $r_{0}, \mathrm{R}_{i}, i=0,1, \ldots, 7$, и не зависящей от $\left(\nu^{\mathrm{I}}, \gamma^{\mathrm{I}}, \rho^{\mathrm{I}}, k^{\mathrm{I}}, k_{1}^{\mathrm{I}}, f^{\mathrm{I}}, u_{0}^{\mathrm{I}}, z^{\mathrm{I}}\right),\left(\nu^{\mathrm{II}}, \gamma^{\mathrm{II}}, \rho^{\mathrm{II}}, k^{\mathrm{II}}, k_{1}^{\mathrm{II}}, f^{\mathrm{II}}, u_{0}^{\mathrm{II}}, z^{\mathrm{II}}\right)$. Учитывая этот факт, нетрудно получить аналог неравенства (3.29) для $w$ и, следовательно, неравенство в определении 3 для $\|w\|_{\varkappa_{T}}$, оценив параметры, где это необходимо, через $r_{0}, \mathrm{R}_{i}, i=0,1, \ldots, 7$, а затем, действуя аналогично получению неравенства (3.33), вывести неравенство в определении 3 для $\|w\| \Xi_{T}$.

Равномерная непрерывность $\Phi$ по Липшицу на ограниченных подмножествах доказана.

Теорема доказана.

ЗАмечАниЕ 1. Очевидно, что единственность в теореме 1 доказана в более широком классе, когда $z \in C\left(0, T ; H^{1}\right) \cap L_{2}\left(0, T ; H^{2}\right), v \in L_{2}\left(0, T ; H^{2}\right), \frac{\partial}{\partial t} v \in$ $L_{2}\left(0, T ; H^{0}\right)$ и уравнение в $(3.7)$ имеет место в пространстве $L_{2}\left(0, T ; H^{-2}\right)$.

ЗАмЕчАНИЕ 2. Если в условии теоремы $1 z \equiv 0$, то мы получаем теорему существования единственного решения задачи Коши для нестохастической системы (1.1) с детерминированными начальными данными $u_{0} \in H^{2}$ и правой частью из класса $g \in L_{2}\left(0, T ; H^{-1}\right)$. Этот результат отличается от результата В. Н. Крупчатникова из [1] тем, что правая часть системы в [1] не зависит от $t$ и принадлежит более узкому классу $g \in H^{-1}$. Дополнительно к результату в [1] в теореме 1 доказана равномерная непрерывность по Липшицу на ограниченных подмножествах оператора, сопоставляющего множеству входящих в задачу параметров решение задачи Коши (3.7), z $\equiv 0$. Таким образом, получена оценка непрерывной зависимости решения детерминированной задачи 
Коши от совокупности параметров $\left(\nu, \gamma, \rho, k, k_{1}\right)$, начальных данных и правой части на конечном отрезке изменения переменной $t$.

Ниже приводится доказательство предложения 1, формулировка которого была приведена в начале $§ 3$.

ДокАЗАтЕЛьство. Единственность. Доказательство единственности полностью аналогично доказательству единственности в теореме 1 , но при этом необходимо оценивать не дифференциальное уравнение, а интегральное в силу определения решения.

Существование. Вначале докажем существование решения системы

$$
\frac{\partial}{\partial t} A_{1} \widehat{z}+\nu A_{2} \widehat{z}+A_{3} \widehat{z}+C_{1} A_{1} \widehat{z}=\exp \left(-C_{1} t\right) \eta, \quad t>0,
$$

где $C_{1}$ - константа $C_{6}$ из оценки (3.24) (рассматриваем случай, когда $C_{1}>0$ ), с начальным условием $\widehat{z}(0)=0$. Будем считать, что определение решения этой системы аналогично определению решения системы (1.1), (1.2), при этом вместо (3.3) будем иметь

$$
\begin{aligned}
A_{1} \widehat{z}(t)+ & \int_{0}^{t}\left(\nu A_{2} \widehat{z}(s)+A_{3} \widehat{z}(s)+C_{1} A_{1} \widehat{z}(s)\right) d s=A_{1} \widehat{z}(0)+\exp \left(-C_{1} t\right) \zeta(t) \\
& +C_{1} \int_{0}^{t} \exp \left(-C_{1} s\right) \zeta(s) d s, \quad t>0 .
\end{aligned}
$$

Заметим, что единственность решения системы (3.45) доказывается полностью аналогично доказательству единственности в теореме 1 , но при этом необходимо оценивать не дифференциальное уравнение, а интегральное в силу определения решения.

Схема доказательства существования решения системы (3.45) с начальным условием $\widehat{z}(0)=0$ аналогична решению упражнения 2.4.4 из работы [24], где доказывается существование решения для системы, похожей на систему (3.45), по переменным $x$ в двумерной ограниченной области или двумерном торе с условием того, что решение и правая часть системы представляют собой бездивергентное векторное поле по переменным $x$.

Рассмотрим $N$-мерное линейное стохастическое уравнение

$$
\frac{\partial}{\partial t} A_{1} z_{N}+\nu A_{2} z_{N}+A_{3} z_{N}+C_{1} A_{1} z_{N}=\exp \left(-C_{1} t\right) \frac{\partial}{\partial t} P_{N} \zeta_{N}, \quad t>0
$$

где $\zeta_{N}=\sum_{i=1}^{2 N(N+2)} b_{i} \beta_{i} E_{i}, z_{N} \in H_{N}$. В силу хорошо известных результатов о существовании и единственности стохастических линейных обыкновенных дифференциальных уравнений (см., например, [16; гл. VIII]) уравнение (3.47) имеет единственное решение, которое удовлетворяет начальному условию $z_{N}(0)=0$, непрерывно по $t$ и согласовано с фильтрацией $F_{t}$. Докажем, что существует подпоследовательность последовательности решений (3.47) с нулевым начальным условием $\left\{z_{N}\right\}_{N=1}^{\infty}$, которая сходится к $\widehat{z}$ в пространстве $\varkappa_{T}$ при любом $T>0$ почти наверное. Для этого вначале получим априорную оценку на $z_{N}$ при помощи формулы Ито и моментного неравенства Дуба, затем, используя похожую технику, докажем, что $z_{N}-z_{M}$ сходится к нулю в полном пространстве $L_{2}\left(\Omega ; \varkappa_{T}\right)$ и предельная функция удовлетворяет (3.46). 
Шаг 1: априорная оценка. В силу формулы Ито (см., например, [30; гл. IV]) получим

$$
\begin{aligned}
\left\|A_{1} z_{N}(t) \mid\right\|_{0}^{2} & =\int_{0}^{t}-2\left\langle\nu A_{2} z_{N}+A_{3} z_{N}+C_{1} A_{1} z_{N}, A_{1} z_{N}\right\rangle d s \\
+ & \frac{1-\exp \left(-2 C_{1} t\right)}{2 C_{1}} \sum_{i=1}^{2 N(N+2)} b_{i}^{2}\left\|P_{N} E_{i} \mid\right\|_{0}^{2}+2 \int_{0}^{t} \exp \left(-C_{1} s\right)\left\langle A_{1} z_{N}, d \zeta_{N}\right\rangle .
\end{aligned}
$$

Взяв от обеих частей равенства математическое ожидание, ввиду (3.23), (3.24), $(3.25)$ и того, что $\left\{b_{i}\right\}_{i=1}^{\infty} \in l_{2}^{+}$, имеем

$$
\sup _{t \in[0, T]} E\left|\left\|A _ { 1 } z _ { N } \left|\left\|_{0}^{2}+2 \nu E \int_{0}^{T}\left|\left\|z_{N} \mid\right\|_{3}^{2} d s \leqslant C_{2}, \quad N=1,2, \ldots .\right.\right.\right.\right.\right.
$$

Везде ниже в шаге 1 считаем, что $C_{i} \geqslant 0, i \geqslant 2,-$ константы, не зависящие от $z_{N}$.

В силу леммы 2 из [16; гл. IV,$\S 4]$ при $h(t)=|t|,[31 ;$ гл. II, § 1], определения 14 из [16; гл. I, §22] и упражнения 28 к [16; гл. I], непрерывности вложения $h^{1}$ в $h^{0}$, оценок (3.26) и (3.49) существует модификация случайного процесса

$$
\left|\int_{0}^{t} \exp \left(-C_{1} s\right)\left\langle A_{1} z_{N}, E_{i}\right\rangle d \beta_{i}\right|,
$$

которая является непрерывным субмартингалом, $i=1,2, \ldots, 2 N(N+2)$. Тогда из моментного неравенства Дуба для непрерывных справа субмартингалов (см., например, [18; гл. II, §5, и гл. IV, §7, теорема 4.7.2]) и неравенства Коши-Буняковского для пространства $\mathbb{R}^{2 N(N+2)}$ вытекает

$$
\begin{aligned}
& E \sup _{t \in[0, T]}\left|\int_{0}^{t} \exp \left(-C_{1} s\right)\left\langle A_{1} z_{N}, d \zeta_{N}\right\rangle\right| \\
& \quad \leqslant \sum_{i=1}^{2 N(N+2)} b_{i} E \sup _{t \in[0, T]}\left|\int_{0}^{t} \exp \left(-C_{1} s\right)\left\langle A_{1} z_{N}, E_{i}\right\rangle d \beta_{i}\right| \\
& \quad \leqslant\left(\sum_{i=1}^{2 N(N+2)} b_{i}^{2}\right)^{1 / 2}\left(\sum_{i=1}^{2 N(N+2)} E \sup _{t \in[0, T]}\left|\int_{0}^{t} \exp \left(-C_{1} s\right)\left\langle A_{1} z_{N}, E_{i}\right\rangle d \beta_{i}\right|^{2}\right)^{1 / 2} \\
& \quad \leqslant 2\left(b \sum_{i=1}^{2 N(N+2)} \sup _{t \in[0, T]} E\left|\int_{0}^{t} \exp \left(-C_{1} s\right)\left\langle A_{1} z_{N}, E_{i}\right\rangle d \beta_{i}\right|^{2}\right)^{1 / 2},
\end{aligned}
$$

где $b=\left\|\left\{b_{i}\right\}_{i=1}^{\infty}\right\|_{l_{2}}$. Ввиду неравенства Бесселя, непрерывности вложения $h^{1}$ в $h^{0}$ и изометрии Ито (см., например, [31; гл. II, $\S 1$, предложение 1.1, (II), формула (1.12)]) имеем

$$
\begin{aligned}
& E \sup _{t \in[0, T]}\left|\int_{0}^{t} \exp \left(-C_{1} s\right)\left\langle A_{1} z_{N}, d \zeta_{N}\right\rangle\right| \\
& \quad \leqslant 2\left(b \sum_{i=1}^{2 N(N+2)} \sup _{t \in[0, T]} E \int_{0}^{t} \exp \left(-2 C_{1} s\right)\left\langle A_{1} z_{N}, E_{i}\right\rangle^{2} d s\right)^{1 / 2}
\end{aligned}
$$




$$
\begin{aligned}
& \leqslant 2\left(b \sum_{i=1}^{2 N(N+2)} E \int_{0}^{T}\left\langle A_{1} z_{N}, E_{i}\right\rangle^{2} d s\right)^{1 / 2}=2\left(b E \int_{0}^{T} \sum_{i=1}^{2 N(N+2)}\left\langle A_{1} z_{N}, E_{i}\right\rangle^{2} d s\right)^{1 / 2} \\
& \leqslant 2\left(b E \int_{0}^{T}\left|\left\|A_{1} z_{N} \mid\right\|_{0}^{2} d s\right)^{1 / 2} \leqslant C_{3}\left(b E \int_{0}^{T}\left\|A_{1} z_{N} \mid\right\|_{1}^{2} d s\right)^{1 / 2} .\right.
\end{aligned}
$$

Отсюда и из $(3.26),(3.49)$ получим

$$
E \sup _{t \in[0, T]}\left|\int_{0}^{t} \exp \left(-C_{1} s\right)\left\langle A_{1} z_{N}, d \zeta_{N}\right\rangle\right| \leqslant C_{4}
$$

В силу (3.23)-(3.25), (3.48)-(3.50) имеем

$$
E \sup _{t \in[0, T]}\left\|A_{1} z_{N}(t)\right\|_{0}^{2} \leqslant C_{2}+2 C_{4} .
$$

Следовательно, из (3.25) и (3.49) вытекает априорная оценка

$$
\left\|z_{N}(t)\right\|_{L_{2}\left(\Omega ; \varkappa_{T}\right)}^{2}=E \sup _{t \in[0, T]}\left|\left\|z _ { N } ( t ) \left|\left\|_{2}^{2}+E \int_{0}^{T}\left|\left\|z_{N} \mid\right\|_{3}^{2} d s \leqslant C_{2}+2 C_{4}+\frac{1}{2 \nu} C_{2} .\right.\right.\right.\right.\right.
$$

Шаг 2: сходимость. Докажем, что существует подпоследовательность последовательности $\left\{z_{N}\right\}_{N=1}^{\infty}$, сходящаяся в пространстве $\varkappa_{T}$ почти наверное, и предельная функция совпадает с $\widehat{z}$.

Рассмотрим разность $z_{M N}=z_{N}-z_{M}, M<N$. Очевидно, что $z_{M N}(0)=0$. В силу свойств ортогонального проектора $P_{N} P_{M}=P_{M}$ и $P_{N}^{2}=P_{N}$ (cм., например, [27; гл. VII, §2]) получим

$$
\frac{\partial}{\partial t} A_{1} z_{M N}+\nu A_{2} z_{M N}+A_{3} z_{M N}+C_{1} A_{1} z_{M N}=\exp \left(-C_{1} t\right) \frac{\partial}{\partial t} P_{N} \zeta_{M N}, \quad t>0,
$$
где

$$
\zeta_{M N}=\sum_{i=1}^{2 M(M+2)} b_{i} \beta_{i}\left(P_{N}-P_{M}\right) E_{i}+\sum_{i=2 M(M+2)+1}^{2 N(N+2)} b_{i} \beta_{i} P_{N} E_{i} .
$$

Ввиду формулы Ито (см., например, [30; гл. IV])

$$
\begin{array}{r}
\left\|A_{1} z_{M N}(t) \mid\right\|_{0}^{2}=\int_{0}^{t}-2\left\langle\nu A_{2} z_{M N}+A_{3} z_{M N}+C_{1} A_{1} z_{M N}, A_{1} z_{M N}\right\rangle d s \\
+\frac{1-\exp \left(-2 C_{1} t\right)}{2 C_{1}} F_{M N}+2 \int_{0}^{t} \exp \left(-C_{1} s\right)\left\langle A_{1} z_{M N}, d \zeta_{M N}\right\rangle,
\end{array}
$$

где

$$
F_{M N}=\sum_{i=1}^{2 M(M+2)} b_{i}^{2}\left\|\left(P_{N}-P_{M}\right) E_{i} \mid\right\|_{0}^{2}+\sum_{i=2 M(M+2)+1}^{2 N(N+2)} b_{i}^{2}\left\|P_{N} E_{i}\right\|_{0}^{2} .
$$

Взяв от обеих частей равенства математическое ожидание в (3.52), из (3.23), (3.24) и (3.25) имеем

$\sup _{t \in[0, T]} E\left|\left\|A_{1} z_{M N}\left|\left\|_{0}^{2}+2 \nu E \int_{0}^{T}\left|\left\|z_{M N} \mid\right\|_{3}^{2} d s \leqslant \frac{1}{2 C_{1}} F_{M N}, \quad N=1,2, \ldots, \quad M<N\right.\right.\right.\right.\right.$. 
Действуя аналогично получению оценки (3.51), выводим

$$
E \sup _{t \in[0, T]}\left|\left\|A_{1} z_{M N}\left|\|_{0}^{2} \leqslant \frac{1}{2 C_{1}} F_{M N}+2 E \sup _{t \in[0, T]}\right| \int_{0}^{t} \exp \left(-C_{1} s\right)\left\langle A_{1} z_{M N}, d \zeta_{M N}\right\rangle \mid\right.\right.
$$

Заметим, что $F_{M N} \rightarrow 0$ при $M, N \rightarrow \infty$. Поэтому нетрудно показать, действуя аналогично получению оценки $(3.50)$, что

$$
E \sup _{t \in[0, T]}\left|\int_{0}^{t} \exp \left(-C_{1} s\right)\left\langle A_{1} z_{M N}, d \zeta_{M N}\right\rangle\right| \rightarrow 0 \quad \text { при } \quad M, N \rightarrow \infty .
$$

Тогда из (3.25), (3.53) и (3.54) вытекает

$$
E \sup _{t \in[0, T]}\left|\left\|z _ { M N } ( t ) \left|\left\|_{2}^{2}+E \int_{0}^{T}\right\|\left\|z_{M N} \mid\right\|_{3}^{2} d s \rightarrow 0 \quad \text { при } \quad M, N \rightarrow \infty .\right.\right.\right.
$$

Поэтому фундаментальная последовательность $\left\{z_{N}\right\}_{N=1}^{\infty}$ сходится к некоторой предельной функции $\bar{z}$ в полном пространстве $L_{2}\left(\Omega ; \varkappa_{T}\right)$. Отсюда следует, что существует подпоследовательность $\left\{z_{N_{j}}\right\}_{j=1}^{\infty}$, которая сходится к $\bar{z}$ в пространстве $\varkappa_{T}$ почти наверное (см., например, [17; гл. II, §10, п. 4, теорема 2, формула (12), и п. 5, теорема 5]). Ввиду того, что $T>0$ любое, $\bar{z} \in \varkappa$ почти наверное.

Поскольку по формуле интегрирования по частям (см., например, [30; гл. IV, $\S 1$, теорема 4.1.5])

$\int_{0}^{t} \exp \left(-C_{1} s\right) d P_{N_{j}} \zeta_{N_{j}}(s)=\exp \left(-C_{1} t\right) P_{N_{j}} \zeta_{N_{j}}(t)+C_{1} \int_{0}^{t} \exp \left(-C_{1} s\right) P_{N_{j}} \zeta_{N_{j}}(s) d s$, то в силу (3.47) $z_{N_{j}}$ удовлетворяет уравнению

$$
\begin{aligned}
A_{1} z_{N_{j}}(t) & +\int_{0}^{t}\left(\nu A_{2} z_{N_{j}}(s)+A_{3} z_{N_{j}}(s)+C_{1} A_{1} z_{N_{j}}(s)\right) d s=\exp \left(-C_{1} t\right) P_{N_{j}} \zeta_{N_{j}}(t) \\
& +C_{1} \int_{0}^{t} \exp \left(-C_{1} s\right) P_{N_{j}} \zeta_{N_{j}}(s) d s, \quad 0 \leqslant t \leqslant T
\end{aligned}
$$

где равенство имеет место в пространстве $C\left(0, T ; H^{-1}\right)$ для почти всех $\omega \in \Omega$. Переходя к пределу по норме пространства $C\left(0, T ; H^{-1}\right)$ при $j \rightarrow \infty$ в этом равенстве, учитывая, что $T>0$ любое, получим, что $\bar{z}$ удовлетворяет уравнению (3.46) в пространстве $C\left(\mathbb{R}^{+} ; H^{-1}\right)$ для любого $t>0$ почти наверное. Ввиду того, что все множества $P$-меры нуль $\sigma$-алгебры $F$ содержатся в $F_{0}$ и случайный процесс $z_{N_{j}}(t), t \geqslant 0$, согласован с фильтрацией $\left\{F_{t}\right\}_{t \geqslant 0}, j=1,2, \ldots$, по лемме 6 из [16; гл. I, §5] и [16; гл. I, §4] следует, что случайный процесс $\bar{z}(t)$, $t \geqslant 0$, согласован с фильтрацией $\left\{F_{t}\right\}_{t \geqslant 0}$. Тогда в силу единственности решения системы $(3.45) \bar{z}(t)=\widehat{z}(t)$ для любого $t \geqslant 0$ с вероятностью 1.

Существование решения системы (3.45) доказано. Заметим, что в случае, когда $C_{1}=0$, легко получить из представленного доказательства существование решения системы (3.45) и, следовательно, (3.4).

Пусть $C_{1}>0$. Поскольку в силу формулы Ито (см., например, [30; гл. IV])

$$
\frac{\partial}{\partial t} A_{1}\left(\exp \left(C_{1} t\right) z_{N_{j}}\right)=C_{1} \exp \left(C_{1} t\right) A_{1} z_{N_{j}}+\exp \left(C_{1} t\right) \frac{\partial}{\partial t} A_{1} z_{N_{j}},
$$


то ввиду (3.47)

$$
\frac{\partial}{\partial t} A_{1}\left(\exp \left(C_{1} t\right) z_{N_{j}}\right)+\left(\nu A_{2}+A_{3}\right)\left(\exp \left(C_{1} t\right) z_{N_{j}}\right)=\frac{\partial}{\partial t} P_{N_{j}} \zeta_{N_{j}}, \quad t>0
$$

Тогда при $0 \leqslant t \leqslant T$

$$
A_{1}\left(\exp \left(C_{1} t\right) z_{N_{j}}(t)\right)+\int_{0}^{t}\left(\nu A_{2}+A_{3}\right)\left(\exp \left(C_{1} s\right) z_{N_{j}}(s)\right) d s=P_{N_{j}} \zeta_{N_{j}}(t),
$$

где равенство имеет место в пространстве $C\left(0, T ; H^{-1}\right)$ для почти всех $\omega \in \Omega$. Переходя к пределу при $j \rightarrow \infty$ в этом равенстве, получим, что $\exp \left(C_{1} t\right) \widehat{z}$ удовлетворяет равенству (3.5) для почти всех $\omega \in \Omega$. Следовательно, в силу единственности решения системы $(3.4) z(t)=\exp \left(C_{1} t\right) \widehat{z}(t)$ для любого $t \geqslant 0$ с вероятностью 1.

Существование решения системы (3.4) с начальным условием $z(0)=0$ доказано.

Предложение доказано.

В следующей теореме доказывается результат о существовании единственного решения задачи Коши (1.1), (1.2), (3.1), а также доказывается оценка непрерывной зависимости этого решения от совокупности случайных начальных данных и внешней силы на конечном отрезке изменения переменной $t$.

Теорема 2. І. Для любых $\nu>0, \gamma, \rho, k, k_{1} \geqslant 0,\left\{b_{i}\right\}_{i=1}^{\infty} \in l_{2}^{+}$, случайного векторного поля $f(t), t \geqslant 0$, согласованного с фильтрацией $\left\{F_{t}\right\}_{t \geqslant 0}, f \in$ $L_{2, \text { lос }}\left(\mathbb{R}^{+} ; H^{-1}\right)$ почти наверное, и $F_{0}$-измеримого случайного векторного поля $u_{0}, u_{0} \in H^{2}$ почти наверное, система (1.1), (1.2) имеет решение $u(t), t \geqslant 0$, удовлетворяющее начальному условию (3.1) почти наверное.

II. Это решение единственно, т.е. если $\widetilde{u}(t)$ - другое решение системь (1.1), (1.2), удовлетворяющее начальному условию (3.1) почти наверное, то $u(t)=\widetilde{u}(t)$ для любого $t \geqslant 0$ с вероятностъю 1 .

Обозначим через $M_{f}$ множество случайных векторных полей, определенных при $t \geqslant 0$, согласованных $c\left\{F_{t}\right\}_{t \geqslant 0}$, принадлежащих почти наверное пространству $L_{2, \operatorname{loc}}\left(\mathbb{R}^{+} ; H^{-1}\right)$, и через $M_{u_{0}}$ множество $F_{0}$-измеримых случайных векторных полей, принадлежащих почти наверное пространству $\mathrm{H}^{2}$. Пусть $u\left(t, x, \omega, f, u_{0}\right)$ - решение задачи Коши (1.1), (1.2), (3.1) со случайньми внешней силой $f$ и начальным данным $u_{0}$.

III. Для любых $T>0$ и случайных величин $\mathrm{R}_{1}(\omega), \mathrm{R}_{2}(\omega) \geqslant 0$ существует такая случайная величина $C\left(\omega, T, \mathrm{R}_{1}(\omega), \mathrm{R}_{2}(\omega)\right) \geqslant 0$, что почти наверное имеет место оченка

$$
\begin{aligned}
& \left\|u\left(t, x, \omega, f^{\mathrm{I}}, u_{0}^{\mathrm{I}}\right)-u\left(t, x, \omega, f^{\mathrm{II}}, u_{0}^{\mathrm{II}}\right)\right\|_{\varkappa_{T}} \\
& \quad \leqslant C(\omega)\left(\left\|f^{\mathrm{I}}(t, x, \omega)-f^{\mathrm{II}}(t, x, \omega)\right\|_{L_{2}\left(0, T ; H^{-1}\right)}+\left\|u_{0}^{\mathrm{I}}(x, \omega)-u_{0}^{\mathrm{II}}(x, \omega)\right\| \|_{2}\right),
\end{aligned}
$$

где $f^{\mathrm{I}}, f^{\mathrm{II}} \in M_{f},\left\|f^{\mathrm{I}}\right\|_{L_{2}\left(0, T ; H^{-1}\right)},\left\|f^{\mathrm{II}}\right\|_{L_{2}\left(0, T ; H^{-1}\right)} \leqslant \mathrm{R}_{1}$ почти наверное, $u_{0}^{\mathrm{I}}, u_{0}^{\mathrm{II}} \in$ $M_{u_{0}},||\left|u_{0}^{\mathrm{I}}\right|\left\|_{2},\left|\left\|u_{0}^{\mathrm{II}} \mid\right\|_{2} \leqslant \mathrm{R}_{2}\right.\right.$ почти наверное - произвольные элементы соответствующих множеств, $C$ не зависит от $f^{\mathrm{I}}, f^{\mathrm{II}}, u_{0}^{\mathrm{I}}, u_{0}^{\mathrm{II}}$. 
ДокАзАтельство. І. Схема доказательства существования аналогична схеме доказательства теоремы 2.4.6 из работы [24], где доказывается существование решения для уравнения Навье-Стокса с правой частью (1.2) по переменным $x$ в двумерной ограниченной области или двумерном торе с условием того, что решение и правая часть системы представляют собой бездивергентное векторное поле по переменным $x$.

Пусть $z(t)$ - решение системы (3.4) с начальным условием $z(0)=0$ (см. предложение 1$)$. Определим $v \in \varkappa$ на $\Omega$ следующим образом: $v(t)$ - решение задачи Коши (3.7) (см. теорему 1) для $\omega \in \Omega_{*}$, где $\Omega_{*}$ определено в $(3.6)$, и $v(t) \equiv 0$ для $\omega \in \Omega \backslash \Omega_{*}$. Тогда из свойств измеримых отображений, согласованности случайных процессов $f(t), t \geqslant 0$, и $z(t), t \geqslant 0$, с фильтрацией $\left\{F_{t}\right\}_{t \geqslant 0}$ и построения решения задачи Коши (3.7) через аппроксимации вытекает, что случайный процесс $v(t), t \geqslant 0$, согласован с фильтрацией $\left\{F_{t}\right\}_{t \geqslant 0}$. Поэтому в силу предложения 1 и теоремы 1 случайный процесс $u(t)=v(t)+z(t)$ - решение системы (1.1), (1.2), удовлетворяющее начальному условию (3.1) почти наверное.

II. Доказательство единственности полностью аналогично доказательству единственности в теореме 1 , но при этом необходимо оценивать не дифференциальное уравнение, а интегральное в силу определения решения.

III. Этот факт сразу следует из доказательства соответствующего результата для $v$ о равномерной непрерывности по Липшицу оператора, сопоставляющего множеству входящих в задачу параметров решение задачи Коши (3.7).

Теорема доказана.

Из леммы 4.5.3 в [18; гл. IV,$\S 5]$ и того, что $(\Omega, F, P)$ - полное вероятностное пространство, $F_{0}$ содержит все множества $P$-меры нуль $\sigma$-алгебры $F$, вытекает

СледствиЕ. В условиях теоремы 2, п. I решение $u(t)$ задачи Коши (1.1), $(1.2),(3.1)$ является прогрессивно измеримым по отношению к $\left\{F_{t}\right\}_{t \geqslant 0}$ случайным процессом.

ЗАмЕчАниЕ 3. В [4; гл. VI, §1] и [32] рассматривалась немного другая двухслойная модель бароклинной атмосферы на единичной двумерной сфере $S$ с центром в нуле. Система уравнений этой модели после обезразмеривания отличается от системы (1.1) только оператором $A_{3}$. А именно, в [4; гл. VI, §1] и [32] оператор $A_{3}$ имел следующий вид:

$$
\widetilde{A}_{3}=\left(\begin{array}{cc}
-k \Delta & -k \Delta \\
-k \Delta & -\left(k+k_{1}\right) \Delta+\rho
\end{array}\right) .
$$

Проводя аналогичные рассуждения, нетрудно показать, что для такой системы выполнены дословно результаты предложения 1, замечания 1, теорем 1, 2 и следствия.

\section{Список литературы}

[1] V. N. Krupchatnikov, "Global attractors for the Lorentz model on the sphere", Bull. Novosib. Comput. Cent., Ser. Numer. Model. Atmos. Ocean Environ. Stud., 2 (1995), 31-40.

[2] E. N. Lorenz, "Energy and numerical weather prediction", Tellus, 12:4 (1960), 364-373. 
[3] В.Н. Крупчатников, Г. П. Курбаткин, Моделирование крупномасштабной динамики атмосферы. Методъ диагноза общей циркуляиии, ВЦ, Сиб. отд-ние АН СССР, Новосибирск, 1991.

[4] В.П. Дымников, А. Н. Филатов, Основы математической теории климата, ВИНИТИ, М., 1994; англ. пер.: V.P. Dymnikov, A. N. Filatov, Mathematics of climate modeling, Model. Simul. Sci. Eng. Technol., Birkhäuser, Boston, MA, 1997.

[5] Б. В. Пальцев, Сферические функции, МФТИ, М., 2000.

[6] Ю.Н. Скиба, Математические вопросы динамики вязкой баротропной жидкости на вращающейся сфере, ОВМ АН СССР, М., 1989.

[7] В. П. Дымников, Устойчивость и предсказуемость крупномасштабных атмосферных процессов, ИВМ РАН, М., 2007.

[8] М. С. Агранович, “Эллиптические сингулярные интегро-дифференциальные операторы", УМH, 20:5 (1965), 3-120; англ. пер.: M. S. Agranovich, "Elliptic singular integro-differential operators", Russian Math. Surveys, 20:5 (1965), 1-121.

[9] К. Иосида, Функииональный анализ, Мир, М., 1967; пер. с англ.: K. Yosida, Functional analysis, Grundlehren Math. Wiss., 123, Springer-Verlag, BerlinGöttingen-Heidelberg; Academic Press, New York, 1965.

[10] С. Г. Михлин, Проблема минимума квадратичного функиионала, Гостехиздат, M.-Л., 1952; англ. пер.: S. G. Mikhlin, The problem of the minimum of a quadratic functional, Holden-Day, San Francisco-London-Amsterdam, 1965.

[11] С.Г. Михлин, Многомерные сингулярные интегралы и интегралъные уравнения, Физматгиз, М., 1962; англ. пер.: S. G. Mikhlin, Multidimensional singular integrals and integral equations, Pergamon Press, Oxford-London-Edinburgh-New York-Paris-Frankfurt, 1965.

[12] С.Г. Михлин, “Дифференцирование рядов по сферическим функциям", Докл. AH CCCP, 126:2 (1959), 278-279.

[13] С. Г. Михлин, Линейные уравнения в частных производных, Высшая школа, М., 1977.

[14] Ж.-Л. Лионс, Э. Мадженес, Неоднородные граничные задачи и их приложения, Мир, M., 1971; пер. с фр.: J.-L. Lions, E. Magenes, Problèmes aux limites non homogènes et applications, Dunod, Paris, 1968.

[15] Ж.-Л. Лионс, Некоторые методы решения нелинейных краевых задач, Мир, М., 1972; пер. с фр.: J.-L. Lions, Quelques méthodes de résolution des problèmes aux limites non linéaires, Dunod, Gauthier-Villars, Paris, 1969.

[16] А.В. Булинский, А.Н. Ширяев, Теория случайных процессов, Физматлит, М., 2005.

[17] А.Н. Ширяев, Вероятность, т. 1: Элементарная теория вероятностей. Математические основания. Предельные теоремы, МЦНМО, М., 2004.

[18] O. Knill, Probability theory and stochastic processes with applications, Overseas Press, New Delhi, 2009.

[19] G. Da Prato, J. Zabczyk, Stochastic equations in infinite dimensions, Encyclopedia Math. Appl., 44, Cambridge Univ. Press, Cambridge, 1992.

[20] И. М. Гельфанд, Н.Я. Виленкин, Некоторые применения гармонического анализа. Оснащенные гилъбертовы пространства, ГИФМЛ, М., 1961; англ. пер.: I. M. Gel'fand, N. Ya. Vilenkin, Generalized functions. Vol. 4: Applications of harmonic analysis, Academic Press, New York-London, 1964.

[21] Yu. N. Skiba, "Spectral approximation in the numerical stability study of nondivergent viscous flows on a sphere", Numer. Methods Partial Differential Equations, 14:2 (1998), 143-157.

[22] А.Н. Колмогоров, С. В. Фомин, Элементы теории функций и функционального анализа, 7-е изд., Физматлит, М., 2004; англ. пер. 1-го изд.: А. N. Kolmogorov, 
S. V. Fomin, Elements of the theory of functions and functional analysis, v. I, II, Graylock Press, Albany, NY, 1957, 1961.

[23] Ю.Н. Скиба, Об однозначной разрешимости уравнения баротропного вихря вязкой жидкости в классах обобщенных функиий на сфере, Препринт №194, ОВМ AH CCCP, M., 1988.

[24] S. Kuksin, A. Shirikyan, Mathematics of two-dimensional turbulence, Cambridge Univ. Press, Cambridge, 2012.

[25] Р. Темам, Уравнения Навъе-Стокса. Теория и численный анализ, Мир, М., 1981; пер. с англ.: R. Temam, Navier-Stokes equations. Theory and numerical analysis, Stud. Math. Appl., 2, North-Holland, Amsterdam-New York, 1979.

[26] М. С. Агранович, Обобщенные функции, МЦНМО, М., 2008.

[27] Л.А. Люстерник, В. И. Соболев, Элементы функиионального анализа, Наука, M., 1965; англ. пер.: L. A. Lusternik, V. J. Sobolev, Elements of functional analysis, Hindustan Publ., Delhi, 1971.

[28] А.Ф. Филиппов, Дифференциальные уравнения с разрывной правой частью, Наука, М., 1985; англ. пер.: А. F. Filippov, Differential equations with discontinuous right-hand sides, Math. Appl. (Soviet Ser.), 18, Kluwer Acad. Publ., Dordrecht, 1988.

[29] Г. В. Демиденко, Введение в теорию соболевских пространств, Учебное пособие, Изд-во Новосиб. ун-та, Новосибирск, 1995.

[30] Б. Оксендаль, Стохастические дифференииальные уравнения. Введение в теорию и приложения, Мир, М., 2003; пер. с англ.: В. Øksendal, Stochastic differential equations, Universitext, Springer-Verlag, Berlin, 2003.

[31] Н. Икэда, С. Ватанабэ, Стохастические дифференциальные уравнения и диффузионные прочессы, Наука, М., 1986; пер. с англ.: N. Ikeda, Sh. Watanabe, Stochastic differential equations and diffusion processes, North-Holland Math. Library, 24, North-Holland, Amsterdam-Oxford-New York, 1981.

[32] А. С. Горелов, "Размерность аттрактора двуслойной бароклинной модели", Докл. PAH, 342:1 (1995), 101-104.

Ю. Ю. Клевцова (Yu. Yu. Klevtsova)

Сибирский региональный научно-исследовательский гидрометеорологический институт, г. Новосибирск

E-mail: yy_klevtsova@ngs.ru
Поступила в редакцию 13.05 .2011 и 26.04 .2012 